\title{
Central instanton production
}

\author{
V. A. Khoze ${ }^{1,2, *}$ V. V. Khoze, ${ }^{2, \dagger}$ D. L. Milne $\oplus^{2, *}$ and M. G. Ryskin $\oplus^{1,2, \S}$ \\ ${ }^{1}$ Petersburg Nuclear Physics Institute, NRC Kurchatov Institute, Gatchina, St. Petersburg 188300, Russia \\ ${ }^{2}$ Department of Physics, Institute for Particle Physics Phenomenology, Durham University, \\ Durham DH1 3LE, United Kingdom
}

(Received 19 November 2021; accepted 24 January 2022; published 17 February 2022)

\begin{abstract}
We study the central production of QCD instantons at hadron colliders in events with two large rapidity gaps. These gaps in rapidity are formed by either Pomeron or photon exchanges or a combination of the two. The $k_{T}$-factorization formalism is used to reduce the factorization scale dependence. We compute for the first time the relevant differential cross sections for a complete set of central instanton production processes at the LHC, including gluon-induced and quark-induced amplitudes, and also show that the largest contribution comes from processes with Pomeron exchanges where a single gluon from each Pomeron couples to the instanton.
\end{abstract}

DOI: $10.1103 /$ PhysRevD.105.036008

\section{INTRODUCTION}

Instantons are nonperturbative field configurations that describe semiclassical transitions between topologically inequivalent vacuum sectors in QCD. Instanton solutions [1] have attracted a lot of interest over the years [2-6], but so far they have not been observed experimentally in any particle physics settings.

The possibility to observe instantons in inelastic protonproton collisions at hadron colliders was considered in [7] and more recently in [8-10]. Instanton processes have large production cross sections at small center-of-mass partonic energies [8], but discovering them at hadron colliders remains challenging [9]. It was shown in [11] that diffractive events with larger rapidity gaps in the final state can provide better conditions for instanton searches, since in this case the soft QCD background caused by multiple parton interactions is suppressed.

In the present paper we consider processes with central instanton production where the secondaries coming from the instanton are separated from the incoming protons (or the proton dissociation products) by two large rapidity gaps (LRGs). Strong interactions across each LRG are conventionally described by a Pomeron emitted in the $t$-channel; see Fig. 1. This kinematic setup implies that we consider

\footnotetext{
*v.a.khoze@durham.ac.uk

†valya.khoze@durham.ac.uk

*daniel.1.milne@durham.ac.uk

${ }^{\S}$ ryskin@thd.pnpi.spb.ru
}

Published by the American Physical Society under the terms of the Creative Commons Attribution 4.0 International license. Further distribution of this work must maintain attribution to the author(s) and the published article's title, journal citation, and DOI. Funded by SCOAP. processes where the instanton was produced in a PomeronPomeron collision, $\mathbb{P P} \rightarrow$ Instanton $\rightarrow X$, where the two Pomerons, $\mathbb{P} \mathbb{P}$, were emitted from the two initial protons, $p p$.

Our approach is to search for instantons in the central exclusive production,

$$
p p \rightarrow p+\mathbb{P}+\mathbb{P}+p \rightarrow p+X+p,
$$

where the initial state protons survive into the final state. The main advantage of such central instanton production processes is a relatively low Pomeron-Pomeron colliding energy which does not allow for a large multiplicity of the background underlying events. Moreover, in this energy range, the central detector becomes almost hermetic (close to $4 \pi$ ) for the Pomeron-Pomeron secondaries and only a small part of the finally produced hadron will avoid detection. ${ }^{1}$ Note that we can consider tagging forwardgoing protons (one or both of them) using dedicated forward proton detectors [14-17]. In particular in the case of the process (1.1) detecting two outgoing protons would allow one to place an upper limit on the instanton mass. New opportunities would be opened with the proposed development of the CMS precision proton spectrometer for the high luminosity LHC [18] which could allow one to

\footnotetext{
${ }^{1}$ The idea to observe instantons in a 2-Pomeron collision was first put forward in [13] in the context of Pomeron collisions at very low invariant mass, $2<M<5 \mathrm{GeV}$ with a roughly isotropic distribution of secondaries. In the present paper we consider instead the central instanton production at larger invariant masses $M \gtrsim 25 \mathrm{GeV}$ where QCD instantons are under much better theoretical control. Of course, the expected instanton production cross section gets smaller at higher instanton masses which nevertheless should be reachable in LHC collisions.
} 


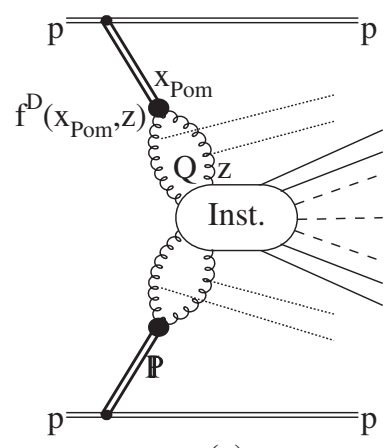

(a)

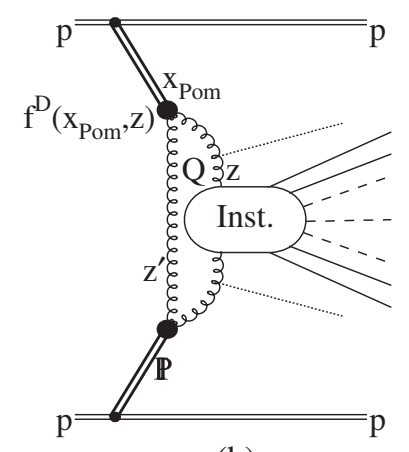

(b)
FIG. 1. Semiexclusive instanton production in a central diffractive process with two LRGs. (a) $(g g)+(g g) \rightarrow$ Instanton subprocess; (b) gluon-gluon fusion subprocess similar to the Durham model [12]. The Pomeron exchange is shown as a thick double line. Solid and dashed lines to the right of the instanton blob denote gluon and quark jets while the dotted lines indicate the possibility of soft gluon emission off the incoming partons.

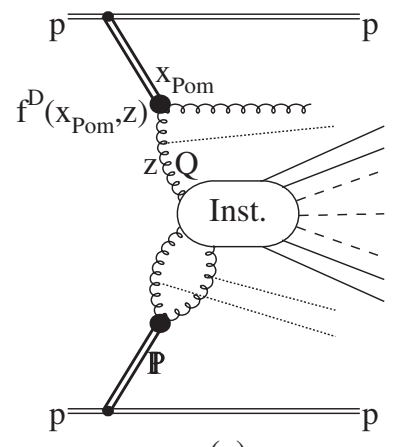

(a)

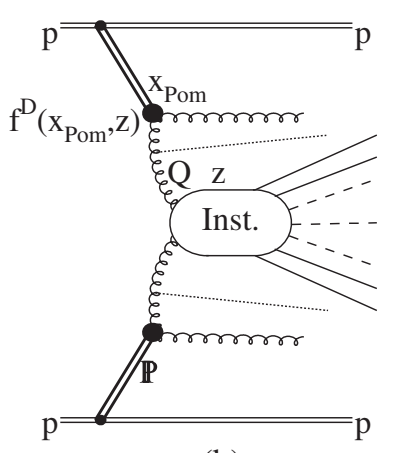

(b)
FIG. 2. Instanton production in central diffractive processes with two LRGs and (a) one or (b) two spectator jets. Pomerons are represented by thick double lines. Solid and dashed lines denote the gluon and quark jets, and the dotted lines indicate the possibility of soft gluon emission off the incoming partons. cover the missing mass range starting from $100 \mathrm{GeV}$ (or even $43 \mathrm{GeV}$ after further modifications).

Since the QCD Pomeron is usually treated as a twogluon color-singlet state, in Sec. II we calculate the amplitude of the interaction of these two gluons (correlated both in color and in their helicities) with the instanton and the cross section of the instanton production in the processes of gluon plus gluon pair, $g+(g g) \rightarrow$ Instanton, and two gluon pair, $(g g)+(g g) \rightarrow$ Instanton, fusion.

Recall that an instanton is not a particle of some fixed mass, but an extended object in spacetime, characterized by certain free parameters (instanton collective coordinates): the instanton size $\rho$, instanton position $x_{0}^{\mu}$, and the orientation in color and Lorentz space. In terms of Feynman diagrams the instanton acts as a multigluon vertex (or more precisely a set of multigluon vertices, each with a different number of gluon legs). Besides this any instanton vertex creates one pair of light quarks of each flavor, $f\left(m_{f}<1 / \rho\right)$. We emphasize that the number of gluons emitted by a particular instanton is not fixed. For this reason we cannot consider the pure exclusive instanton production. The process can be accompanied by the radiation of additional partons, and it is not clear how to separate these additional gluons (or quarks) from those emitted by the instanton. Therefore in Sec. III we compare the cross sections of a few different processes:

(i) pure exclusive instanton production $\mathbb{P}+\mathbb{P} \rightarrow$ $(g g)+(g g) \rightarrow$ Inst,

(ii) instanton plus bremsstrahlung gluons $\mathbb{P}+\mathbb{P} \rightarrow$ $(g g)+(g g) \rightarrow$ Inst $+n g$

(iii) instanton plus a spectator gluon $g_{s}$, such as

$$
\begin{aligned}
& \mathbb{P}+\mathbb{P} \rightarrow(g g)+g+g_{s} \rightarrow \text { Inst }+g_{s}, \\
& \mathbb{P}+\mathbb{P} \rightarrow g+g_{s}+g+g_{s} \rightarrow g_{s}+\text { Inst }+g_{s} .
\end{aligned}
$$

(iv) Furthermore, large rapidity gaps can also be formed by a photon exchange,

$$
\begin{aligned}
& \gamma+\mathbb{P} \rightarrow q \bar{q}_{s}+(g g) \rightarrow \bar{q}_{s}+\text { Inst, } \\
& \gamma+\gamma \rightarrow q \bar{q}_{s}+q_{s} \bar{q} \rightarrow \bar{q}_{s}+\text { Inst }+q_{s} .
\end{aligned}
$$

The diagrams describing all these cases are shown in Figs. 1, 2 , and 3 .

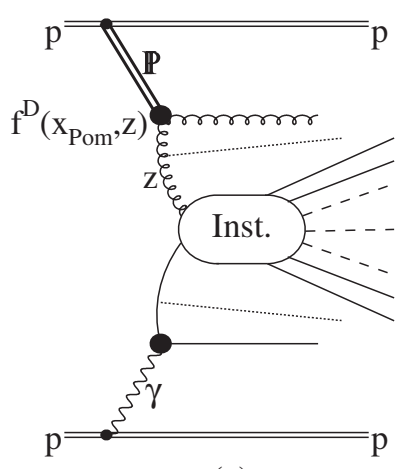

(a)

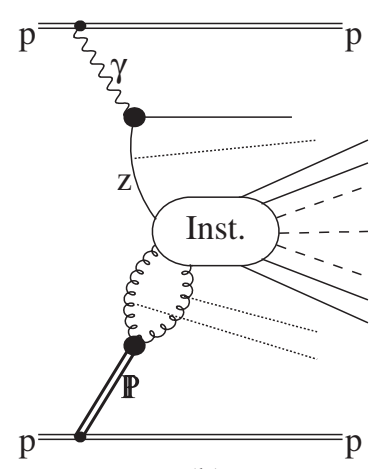

(b)

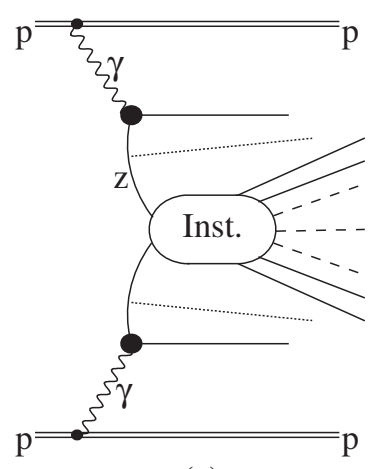

(c)

FIG. 3. Instanton (plus spectators) production in (a), (b) photon-Pomeron and (c) photon-photon collisions. Notation is the same as in Figs. 1 and 2. 
The photon-exchange initiated central instanton production processes corresponding to Fig. 3 will be discussed in Sec. IV.

In Sec. $V$ we present numerical estimates for the processes listed above and also discuss the possibility to select events with a large $p_{T}$ jet formed by the gluon or quark spectator. The large transverse momentum, $p_{T}$, of the jet is compensated by the instanton. This rejects contributions of large size instantons and allows one to search for small size instantons in cleaner theoretical settings. We present our conclusions in Sec. VI.

\section{GLUONS IN THE INSTANTON BACKGROUND}

Here we collect some useful formulas for the sum over polarizations and color indices of the initial state gluons computed on the instanton configuration. These expressions will be relevant for computing elementary partonlevel instanton cross sections for different sets of initial gluon configurations considered in this paper. Specifically, as can be inferred from Figs. 1(a), 1(b), 2(a), and 2(b), the desired hadronic cross sections $\sigma$ for these processes rely on the knowledge of the following parton-level instanton cross sections $\hat{\sigma}$,

$$
\begin{gathered}
\sigma^{(1 a)}: \hat{\sigma}_{(g g)+(g g) \rightarrow \text { Inst }}, \\
\sigma^{(2 a)}: \hat{\sigma}_{g+(g g) \rightarrow \text { Inst }}, \\
\sigma^{(1 b)} \& \sigma^{(2 b)}: \hat{\sigma}_{g+g \rightarrow \text { Inst }} .
\end{gathered}
$$

Each elementary instanton cross section $\hat{\sigma}$ is obtained via the optical theorem by computing the imaginary part of the forward scattering amplitude in an instanton-anti-instanton background. For example, for the instanton cross section $\hat{\sigma}_{g+g \rightarrow \text { Inst }}$ with two gluons in the initial state, we have

$$
\hat{\sigma}_{g+g \rightarrow \operatorname{Inst}}(\hat{s})=\frac{1}{\hat{s}} \operatorname{Im} \mathcal{A}_{4}^{I \bar{I}}\left(p_{1}, p_{2},-p_{1},-p_{2}\right),
$$

where $\sqrt{\hat{s}}=\sqrt{\left(p_{1}+p_{2}\right)^{2}}$ is the partonic centre-of-mass (c.m.) energy, and the forward elastic scattering amplitude reads $[8,9]$

$$
\begin{aligned}
\mathcal{A}_{4}^{I \bar{I}}= & \int_{0}^{\infty} d \rho \int_{0}^{\infty} d \bar{\rho} \int d^{4} R \\
& \times \int d u D(\rho) D(\bar{\rho}) e^{-S_{I \bar{I}}-\frac{\alpha_{S}}{16 \pi}\left(\rho^{2}+\bar{\rho}^{2}\right) \hat{s} \log \frac{\hat{s}}{\mu_{r}^{2}}} \\
& \times \mathcal{K}_{\mathrm{ferm}} A_{\mathrm{LSZ}}^{\text {inst }}\left(p_{1}\right) A_{\mathrm{LSZ}}^{\text {inst }}\left(p_{2}\right) A_{\mathrm{LSZ}}^{\overline{\mathrm{inst}}}\left(-p_{1}\right) A_{\mathrm{LSZ}}^{\overline{\text { inst }}}\left(-p_{2}\right) .
\end{aligned}
$$

In the expression above we integrate over all collective coordinates: $\rho$ and $\bar{\rho}$ are the instanton $(I)$ and anti-instanton
$(\bar{I})$ scale sizes, $R_{\mu}=\left(R_{0}, \vec{R}\right)$ is the separation between the instanton and anti-instanton positions, and $u$ is the $3 \times 3$ matrix of relative $I \bar{I}$ orientations in the $S U(3)$ color space. $D(\rho)$ and $D(\bar{\rho})$ represent the known instanton and antiinstanton densities, $S_{I \bar{I}}$ is the Euclidean action of the instanton-anti-instanton configuration, and $\mathcal{K}_{\text {ferm }}$ is another known factor accounting for the presence of quarks in the theory.

The term $-\frac{\alpha_{s}}{16 \pi}\left(\rho^{2}+\bar{\rho}^{2}\right) \hat{s} \log \frac{\hat{s}}{\mu_{r}^{2}}$ appearing in the exponent represents the leading-order quantum effects from radiative exchanges between the hard initial states in the instanton background computed in [19].

The two-gluon initial state is represented on the righthand side of (2.5) by the field insertions of $A_{\mathrm{LSZ}}^{\mathrm{inst}}\left(p_{1}\right)$ and $A_{\mathrm{LSZ}}^{\mathrm{inst}}\left(p_{2}\right)$, and the similar factor involving the antiinstanton fields appearing in (2.5) represents the two-gluon final state of the forward elastic scattering amplitude.

We can now write down a single expression to represent all elementary instanton cross sections in (2.1)-(2.3) as

$$
\begin{aligned}
\hat{\sigma}_{|i n\rangle \rightarrow \operatorname{Inst}}\left(\hat{s}, Q_{1}^{2}, Q_{2}^{2}\right)= & \frac{1}{\hat{s}} \operatorname{Im} \int_{0}^{\infty} d \rho \int_{0}^{\infty} d \bar{\rho} \int d^{4} R \\
& \times \int d u D(\rho) D(\bar{\rho}) \mathcal{K}_{\text {ferm }} \\
& \times\langle\cdots\rangle_{|i n\rangle} e^{-S_{I \bar{I}}-\frac{\alpha_{s}}{16 \pi}\left(\rho^{2}+\bar{\rho}^{2}\right) \hat{s} \log \frac{\hat{s}}{\mu_{r}^{2}}}
\end{aligned}
$$

The quantity $\langle\cdots\rangle$ on the right-hand side of (2.6) represents the instanton (and anti-instanton) field insertions that correspond to the initial state $|i n\rangle$ (and its conjugate $\langle i n|$ ) in the cross section. The arguments of the instanton cross section $\hat{\sigma}_{|i n\rangle \rightarrow \text { Inst }}$ are the partonic center of mass energy $\hat{s}$ (also referred to as the instanton mass) and the virtualities, $Q_{1}^{2}$ and $Q_{2}^{2}$, of the initial partonic states. In fact, as will become clear a little later, it will often make sense to distinguish between the momenta of the incoming parton states $Q_{1}^{2}, Q_{2}^{2}$ in the forward elastic scattering amplitude (2.6) and the momenta of the outgoing states that we denote $\bar{Q}_{1}^{2}, \bar{Q}_{2}^{2}$, which are no longer assumed to be identical to the incoming momenta. In this case the instanton cross section is a function of five arguments, $\hat{\sigma}_{|i n\rangle \rightarrow \mathrm{Inst}}\left(\hat{s}, Q_{1}^{2}, \bar{Q}_{1}^{2}, Q_{2}^{2}, \bar{Q}_{2}^{2}\right)$.

In Secs. II A-II D we specify the classical field insertions $\langle\cdots\rangle$ for each of the cases in Eqs. (2.1)-(2.3). The expressions for $D(\rho), \mathcal{K}_{\text {ferm }}$, and $S_{I \bar{I}}$ are taken from [8,9] and for completeness are listed in Appendix A.

\section{A. Initial gluons of arbitrary color and polarizations}

An incoming gluon of momentum $p$, helicity $\lambda$, and color $a$ is represented in the path integral for the scattering amplitude by an insertion of the LSZ-reduced instanton configuration $A_{\mathrm{LSZ}}^{\text {ainst }}(p, \lambda)$, which for the on-shell massless gluon reads (see, e.g., [8]) 


$$
\begin{aligned}
A_{\mathrm{LSZ}}^{a \text { inst }}(p, \lambda) & =\lim _{p^{2} \rightarrow 0} p^{2} \epsilon^{\mu}(p, \lambda) A_{\mu}^{a \text { inst }}(p) \\
& =\epsilon^{\mu}(p, \lambda) \bar{\eta}_{\mu \nu}^{a} p_{\nu} \frac{4 i \pi^{2} \rho^{2}}{g} e^{i p \cdot x_{0}} .
\end{aligned}
$$

In the expression above $\epsilon^{\mu}(p, \lambda)$ is the gluon polarization vector and $A_{\mu}^{a \text { inst }}(p)$ is the instanton configuration (in the singular gauge) Fourier transformed into momentum space. The instanton size is $\rho$, the instanton center is $x_{0}$, and $\bar{\eta}_{\mu \nu}^{a}$ are the 't Hooft eta symbols. It is often convenient to work with the instantons in matrix representation, in which case we define $A_{\mu}=A_{\mu}^{a} T^{a}$, where $T^{a}=\lambda^{a} / 2$ are the conventional generators of $S U(3)_{c}$ and only the first three generators contribute since the instanton lives in the $S U(2)$ subgroup of $S U(3)_{c}$. In matrix notation we have

$$
A_{\mathrm{LSZ}}^{\text {inst }}(p, \lambda)=\frac{2 \pi^{2} \rho^{2}}{g} \epsilon^{\mu}(p, \lambda)\left(\sigma_{\mu} \bar{p}-p_{\mu} \mathbb{1}\right) e^{i p \cdot x_{0}},
$$

where $\bar{p}=\bar{\sigma}_{\mu} p^{\mu}$ and $\sigma_{\mu}$ and $\bar{\sigma}_{\mu}$ are the usual sets of four sigma matrices.

To take into account the contribution of a single gluon with the helicity $\lambda$ and color index $a$ we use the insertion

$$
\frac{1}{2} \sum_{\lambda=1,2} \frac{1}{8} \sum_{a=1}^{8} A_{\mathrm{LSZ}}^{a \mathrm{inst}}(p, \lambda) A_{\mathrm{LSZ}}^{a \overline{\mathrm{inst}}}(-p, \lambda)
$$

in the path integral representation for the forward scattering amplitude. This is easiest to evaluate using the matrix representation (2.8) for the instanton field and

$A_{\mathrm{LSZ}}^{\overline{\mathrm{inst}}}(p, \lambda)=\frac{2 \pi^{2} \bar{\rho}^{2}}{g} \epsilon^{\mu}(p, \lambda)\left(u \bar{\sigma}_{\mu} p \bar{u}-p_{\mu} \mathbb{1}\right) e^{i p \cdot \bar{x}_{0}}$

for the anti-instanton, where $u$ denotes the matrix of relative instanton-anti-instanton orientation in color space, and $\bar{\rho}$ and $\bar{x}_{0}$ are the anti-instanton scale size and position. We now evaluate the sum in (2.9) and find (reproducing the result in [20])

$\frac{1}{8} \sum_{\lambda=1,2} \operatorname{Tr}\left(A_{\mathrm{LSZ}}^{\mathrm{inst}}(p, \lambda) A_{\mathrm{LSZ}}^{\overline{\mathrm{inst}}}(-p, \lambda)\right)=\frac{\pi^{3}}{\alpha_{s}}(p \cdot \hat{R})^{2} \rho^{2} \bar{\rho}^{2} e^{i R \cdot p}$.

In deriving this expression we chose the maximally attractive instanton-anti-instanton orientation for which $u$ lives in the $S U(2)$ subgroup of $S U(3)^{2}$ and is given by $u=\sigma^{\mu} \hat{R}_{\mu}$ (where $\hat{R}_{\mu}=R_{\mu} / \sqrt{R^{2}}$ and $R$ is the instantonanti-instanton separation) and used the standard completeness relation for the sum over gluon polarizations,

\footnotetext{
${ }^{2}$ So that in the maximally attractive channel for the instantonanti-instanton potential, both instantons are located in the same $S U(2)$ subgroup.
}

$$
\sum_{\lambda=1,2} \epsilon^{\mu}(p, \lambda) \epsilon^{* \nu}(p, \lambda)=-g_{\perp}^{\mu \nu}
$$

After an analytic continuation to Minkowski space $i R \rightarrow R$ and also allowing for the possibility of virtuality $Q$ in the gluon momentum, $Q^{2}=-p^{2} \ll \hat{s}$, we find

$$
\begin{aligned}
\frac{1}{8} & \sum_{\lambda=1,2} \operatorname{Tr}\left(A_{\mathrm{LSZ}}^{\mathrm{inst}}(p, \lambda) A_{\mathrm{LSZ}}^{\overline{\mathrm{inst}}}(-p, \lambda)\right) \\
= & \frac{\pi^{3}}{4} \frac{1}{\alpha_{s}} \hat{s} \rho^{2} \bar{\rho}^{2} J(Q \rho) J(Q \bar{\rho}) e^{R \cdot p},
\end{aligned}
$$

where $J(Q \rho)$ is a form factor associated with the gluon of virtuality $Q$ and is given by the Bessel function $K_{1}$,

$$
J(x)=x K_{1}(x) .
$$

The form factor interpolates between $J(0)=1$ for the onshell gluon and the exponential $J(x) \rightarrow \sqrt{\frac{\pi x}{2}} e^{-x}$ for the highly virtual one at $x \gg 1$. The formula (2.14) was obtained in [21,22] using the Fourier transformation of the instanton field with a general value of $Q$ and paying careful consideration to preserving gauge invariance in the context of applications to deep inelastic scattering. ${ }^{3}$

In the expression on the right-hand side in (2.13) we also made the substitution $(p \cdot \hat{R})^{2}=\hat{s} / 4$ which follows from the fact that in the center of mass frame the saddlepoint solution for the instanton-anti-instanton separation is along the time direction, $\hat{R}=(1,0,0,0)$, and that for the two-gluon initial state, $p_{0}=\sqrt{\hat{s}} / 2$. The expression (2.13) agrees with the formula used in [8] if the latter is corrected by an overall "normalization" factor of $2 / 3$ and one accounts for the instanton and anti-instanton form factors (2.14).

The contribution of two such initial state gluons with momenta $p_{1}$ and $p_{2}$ gives a factor of

$$
\langle\cdots\rangle_{(g+g)_{Q}}=\left(\frac{\pi^{3}}{4} \frac{1}{\alpha_{s}}\right)^{2}\left(\hat{s} \rho^{2} \bar{\rho}^{2}\right)^{2} J^{2}(Q \rho) J^{2}(Q \bar{\rho}) e^{R \cdot\left(p_{1}+p_{2}\right)}
$$

to the integrand of the expression for the parton-level instanton cross section in (2.3). Here $\hat{s}=2 p_{1} \cdot p_{2}$. Thus for the initial state $|i n\rangle=g+g$ the instanton cross section in (2.3) is computed by substituting ${ }^{4}$ the expression (2.15)

\footnotetext{
${ }^{3}$ Specifically the derivation in [21] concerned the photonquark-antiquark vertex and the associated quark propagators in the instanton background. In our case the consideration would involve the Pomeron-gluon-gluon vertex, but we do not expect that the resulting form factor would be different. In any case, the precise expression is not particularly important as we are concerned here with rather small values of $Q \sim \mathrm{GeV} \ll \sqrt{\hat{s}}$. In Ref. [11] we used the simplified formula $J(x) \simeq e^{-x}$ instead of the more complete form in (2.14).

${ }^{4}$ It is easily seen that (2.15) has the correct dimensions of $\rho^{2} \bar{\rho}^{2}$ as expected for the insertion of four LSZ-reduced gluon fields.
} 
into the integral in Eq. (2.6). The subscript $Q$ displayed in our notation for the $(g+g)_{Q}$ initial state on the left-hand side of (2.15) indicates that both of these initial state gluons have virtualities $Q$.

Combining Eqs. (2.6) and (2.15) gives us the partonlevel instanton cross-section formula that will be used in Sec. III in obtaining the proton-proton cross sections $\sigma^{(2 b)}$ in Eqs. (3.4) and (3.10), and $\sigma^{(1 b)}$ following (3.16).

In the case of $\sigma^{(1 b)}$ it will be important to formally distinguish between the momenta of gluon states in the amplitude and in the complex conjugate amplitude. ${ }^{5}$ Thus we denote the virtualities of the incoming gluons in the instanton background as $Q_{t}$, for the conjugate amplitude these virtualities in the anti-instanton background are denoted as $\bar{Q}_{t}$, and we rewrite (2.15) as

$$
\langle\cdots\rangle_{(g+g)_{Q_{t}, \bar{Q}_{t}}}=\left(\frac{\pi^{3}}{4} \frac{1}{\alpha_{s}}\right)^{2}\left(\hat{s} \rho^{2} \bar{\rho}^{2}\right)^{2} J^{2}(Q \rho) J^{2}(\bar{Q} \bar{\rho}) e^{R \cdot\left(p_{1}+p_{2}\right)} .
$$

\section{B. A gluon pair in a color-singlet state}

We now consider the contribution of a single pair of initial state gluons in a color-singlet state $(g g)$. From one (gg) pair the scattering amplitude receives the contribution

$$
\begin{gathered}
\frac{1}{8} \sum_{a=1}^{3} \frac{1}{2} \sum_{\lambda=1,2} A_{\mathrm{LSZ}}^{a \text { inst }}\left(p_{1}, \lambda\right) A_{\mathrm{LSZ}}^{a \text { inst }}\left(p_{2}, \lambda\right) \\
\quad=\frac{4 \pi^{3}}{16} \frac{1}{\alpha_{s}} \hat{s}_{12} \rho^{4} J^{2}(Q \rho) e^{i x_{0} \cdot\left(p_{1}+p_{2}\right)} .
\end{gathered}
$$

In evaluating the expression on the right-hand side of (2.17) we used the sum over polarization vectors that corresponds to the relevant for us $J_{z}=0$ mode of the gluon pair,

$$
\sum_{\lambda=1,2} \epsilon^{\mu}\left(p_{1}, \lambda\right) \epsilon^{\nu}\left(p_{2}, \lambda\right)=-g^{\mu \nu}+\frac{p_{1}^{\mu} p_{2}^{\nu}}{p_{1} \cdot p_{2}}+\frac{p_{2}^{\mu} p_{1}^{\nu}}{p_{1} \cdot p_{2}}
$$

along with the simplifying relation for the sum of eta symbols,

$$
\sum_{a} \bar{\eta}_{\mu \nu}^{a} \bar{\eta}_{\alpha \beta}^{a}=\delta_{\mu \alpha} \delta_{\nu \beta}-\delta_{\mu \beta} \delta_{\nu \alpha}-\varepsilon_{\mu \nu \alpha \beta} .
$$

This implies

$$
\begin{aligned}
& \sum_{a} \sum_{\lambda} \bar{\eta}_{\mu \nu}^{a} \bar{\eta}_{\alpha \beta}^{a} \epsilon^{\mu}\left(p_{1}, \lambda\right) p_{1}^{\nu} \epsilon^{\alpha}\left(p_{2}, \lambda\right) p_{2}^{\beta} \\
& \quad=\left(\epsilon_{1} \cdot \epsilon_{2}\right)\left(p_{1} \cdot p_{2}\right)-\left(\epsilon_{1} \cdot p_{2}\right)\left(\epsilon_{2} \cdot p_{1}\right) \\
& \quad=-2 p_{1} \cdot p_{2},
\end{aligned}
$$

\footnotetext{
${ }^{5}$ So that we can integrate over the gluon loop momentum in Fig. 1(b) at the level of the amplitude.
}

and Eq. (2.17) follows. Then the contribution to the cross section from such a gluon pair in the color-singlet state amounts to

$$
\left(\frac{\pi^{3}}{4} \frac{1}{\alpha_{s}}\right)^{2}\left(\hat{s}_{12} \rho^{2} \bar{\rho}^{2}\right)^{2} J^{2}(Q \rho) J^{2}(Q \bar{\rho}) e^{R \cdot\left(p_{1}+p_{2}\right)} .
$$

Note that this expression is the same as in (2.15).

When the initial state of the instanton process originates from a Pomeron $\mathbb{P}$, the Pomeron can emit a gluon pair. This gluon pair is in a color-singlet state and also has collinear momenta, $p_{g_{1}}=z p_{\mathbb{P}}$ and $p_{g_{2}}=(1-z) p_{\mathbb{P}}$. For the strictly massless on-shell gluon momenta, the expressions in (2.17) and (2.20) will vanish since for the collinear on-shell gluon momenta $\hat{s}_{12}=0$.

The leading correction to this vanishing result comes from the inclusion of gluon virtualities which in Eqs. (3.11) and (3.14) will be identified with the transverse momentum $Q_{t}^{2}$ of gluons in the $(g g)$ loop. The contribution of these gluon insertions to the cross section is determined by (2.20) with the substitution $\hat{s}_{12}=Q_{t}^{2}$.

When computing the hadronic cross section based on this parton level instanton cross section in Sec. III, it will be important to formally distinguish between the momenta of the incoming $(g g)$ gluon pair in the amplitude and the corresponding $(g g)$ state in the complex conjugate amplitude. ${ }^{6}$ Thus we denote the virtualities of the incoming gluons in the instanton background as $Q_{t}$, and for the conjugate amplitude these virtualities in the anti-instanton background are denoted as $\bar{Q}_{t}$. In summary, for the field insertion relevant to a single gluon pair of states we have

$\langle\cdots\rangle_{(g g)_{Q_{t}, \bar{Q}_{t}}}=\left(\frac{\pi^{3}}{4} \frac{1}{\alpha_{s}}\right)^{2} Q_{t}^{2} \bar{Q}_{t}^{2} \rho^{4} \bar{\rho}^{4} J^{2}\left(Q_{t} \rho\right) J^{2}\left(\bar{Q}_{t} \bar{\rho}\right) e^{R \cdot\left(p_{1}+p_{2}\right)}$.

\section{Three gluons in the initial state $\hat{\boldsymbol{\sigma}}_{g+(g g) \rightarrow \text { Inst }}$}

Here we consider the process shown in Fig. 2(a) where the first proton of momentum $p_{1}$ emits a single gluon $g_{1}$ with momentum $p_{g_{1}}=x_{1} p_{1}$, and the second proton $p_{2}$ emits a Pomeron with momentum $p_{\mathbb{P}}=x_{2} p_{2}$ that subsequently produces two gluons with momenta $p_{g_{2}}=z p_{\mathbb{P}}$ and $p_{g_{3}}=(1-z) p_{\mathbb{P}}$. The instanton process initiated by these three gluons is

$$
g_{1}+\left(g_{2} g_{3}\right) \rightarrow X,
$$

where the brackets indicate that the gluon pair originated from the Pomeron. The corresponding parton-level instanton cross section is $\hat{\sigma}_{g+(g g) \rightarrow \text { Inst }}$.

\footnotetext{
${ }^{6}$ So that we can integrate over the loop momenta of each $(g g)$ state in (3.14) independently.
} 
The corresponding three-gluon insertion factor is given by the product of the expressions in Eqs. (2.21) and (2.13). It reads

$$
\begin{aligned}
\langle\cdots\rangle_{Q_{Q_{1 t}}+(g g)_{Q_{2 t}, \bar{Q}_{2 t}}=} & \left(\frac{\pi^{3}}{4} \frac{1}{\alpha_{s}}\right)^{3} Q_{2 t}^{2} \bar{Q}_{2 t}^{2} \hat{s} \rho^{6} \bar{\rho}^{6} J\left(Q_{1 t} \rho\right) J\left(Q_{1 t} \bar{\rho}\right) \\
& \times J^{2}\left(Q_{2 t} \rho\right) J^{2}\left(\bar{Q}_{2 t} \bar{\rho}\right) e^{R \cdot\left(p_{1}+p_{2}+p_{3}\right)} .
\end{aligned}
$$

Here $\hat{s}=x_{1} x_{2} s_{p p}=M_{\text {inst }}^{2}$; the virtuality of the single gluon $g$ is denoted as $Q_{1 t}$; and the virtualities of the gluon in the $(g g)$ pair are $Q_{2 t}$ for the amplitude and $\bar{Q}_{2 t}$ for the conjugate amplitude. These conventions correspond to what will be used in (3.17) in Sec. III for the protonproton cross section $\sigma^{(2 a)}$.

The overall dimensionality of the expression in (2.23) is $\rho^{3} \bar{\rho}^{3}$, which correctly represents three instanton and three anti-instanton fields in the cross section. The instanton cross section $\hat{\sigma}_{g+(g g) \rightarrow \text { Inst }}$ is then obtained by plugging (2.23) into the integral in Eq. (2.6). In Sec. III we will use this to obtain the proton-proton cross section $\sigma^{(2 a)}$.

For completeness, we should also comment on the function in the exponent in the instanton cross-section integral (2.6), though it will turn out that these additional contributions [see Eq. (2.26) below] will vanish in the relevant for us $z \rightarrow 1$ limit dictated by Eq. (3.13) in Sec. III. The only modifications relevant to the three gluon initial state arise in the term

$$
\exp \left(-\frac{\alpha_{s}}{16 \pi}\left(\rho^{2}+\bar{\rho}^{2}\right) \hat{s} \log \hat{s}\right),
$$

which describes the radiative exchanges between the two initial gluons, and should now be modified as follows. For our present case of three gluons in the initial state, we should account for the interactions between $g_{1}$ and $g_{2}$, and between $g_{1}$ and $g_{3}$ (there are no new effects for $g_{2}-g_{3}$ exchanges, as these gluons are collinear). We have

$\exp \left(-\frac{\alpha_{s}}{16 \pi}\left(\rho^{2}+\bar{\rho}^{2}\right)\left(\hat{s}_{13} \log \hat{s}_{13}+\hat{s}_{12} \log \hat{s}_{12}\right)\right)$.

Comparing the expressions (2.24) and (2.25) gives the overall relative factor

$\mathcal{R}=\exp \left(-\frac{\alpha_{s}}{16 \pi}\left(\rho^{2}+\bar{\rho}^{2}\right) \hat{s}(z \log z+(1-z) \log (1-z))\right)$,

which should be included in the integral in Eq. (2.6).

\section{Four gluons in the initial state $\hat{\sigma}_{(g g)+(g g) \rightarrow \text { Inst }}$}

We can now repeat the analogous steps for the subprocess in Fig. 1(a) with two gluon pairs in the initial state,

$$
(g g)_{1}+(g g)_{2} \rightarrow X
$$

The relevant field insertion in the integral for the cross section is given by the product of the two insertions in (2.21),

$$
\langle\cdots\rangle_{(g g)_{1}+(g g)_{2}}=\langle\cdots\rangle_{(g g)_{Q_{1 t}, \bar{Q}_{1 t}}} \cdot\langle\cdots\rangle_{(g g)_{Q_{2 t} \bar{Q}_{2 t}}},
$$

with the virtualities of the first $(g g)$ pair set to $Q_{1 t}$ in the amplitude and $\bar{Q}_{1 t}$ in the conjugate amplitude, and for the second $(g g)$ pair we use $Q_{2 t}$ and $\bar{Q}_{2 t}$ for the amplitude and conjugate amplitude, respectively.

The parton-level instanton cross section $\hat{\sigma}_{(g g)+(g g) \rightarrow \text { Inst }} \times$ $\left(\hat{s}, Q_{1 t}^{2}, \bar{Q}_{1 t}^{2}, Q_{2 t}^{2}, \bar{Q}_{2 t}^{2}\right)$ for the process (2.27) is obtained by plugging in the expression for the field insertions (2.28) into the integral (2.6). In Sec. III we will use this to obtain the proton-proton cross section $\sigma^{(2 a)}$ in Eq. (3.14).

\section{INSTANTON PRODUCTION IN POMERON COLLISIONS}

\section{A. Partons created by a Pomeron}

The presence of an LRG on a large rapidity interval is conventionally described by a Pomeron exchange in the $t$-channel. Pomerons do not interact with the instanton directly; they first produce partons (gluons or quarks) which then couple to the instanton. The spectra of these partons are described by the diffractive parton distribution functions (dPDF) denoted as $f_{i}^{D}\left(x_{\mathbb{P}}, z, \mu ; t\right)$ where $x_{\mathbb{P}}$ is the proton momentum fraction carried by the Pomeron, $z$ is the Pomeron momentum fraction carried by the parton $i, \mu$ is the scale at which the dPDF of interest enters (measured), and $t$ is the square of the momentum transferred through the Pomeron: $i=g, q_{f}$.

In our calculations we will use the diffractive parton distributions given by fit $\mathrm{B}$ of the $\mathrm{H} 1$ group [23] which reasonably well describe the deep inelastic events with LRGs observed at HERA. The dPDF in this analysis was calculated as

$$
f_{i}^{D, t}\left(x_{\mathbb{P}}, z, \mu^{2} ; t\right)=f_{\mathbb{P} / p}\left(x_{\mathbb{P}}, t\right) f_{i}\left(z=x / x_{\mathbb{P}}, \mu\right) .
$$

Here

$$
f_{\mathbb{P} / p}\left(x_{\mathbb{P}}, t\right)=g_{N}^{2} \frac{e^{B_{\mathbb{P}} t}}{x_{\mathbb{P}}^{2 \alpha_{\mathbb{P}}(t)-1}}
$$

is the Pomeron flux written in terms of the simple Regge pole parametrization, $\alpha_{\mathbb{P}}(t)$ is the Pomeron pole trajectory, $g_{N}$ is the proton-Pomeron coupling, and $B_{\mathbb{P}} / 2$ is its slope. The parton distributions inside the Pomeron, $f_{i}(z, \mu)$, were parametrized at the initial value of $\mu^{2}=\mu_{0}^{2}=2.5 \mathrm{GeV}^{2}$ and then evolved via the QCDNUM package [24]. 
The distribution function $f_{i}^{D, t}\left(x_{\mathbb{P}}, z, \mu^{2} ; t\right)$ in (3.1) is the probability to find a parton $i$ in the Pomeron that has a fixed value of $t$. We also define the more conventional $t$-independent diffractive PDF by integrating the expression in (3.1) over $t$,

$$
f_{i}^{D}\left(x_{\mathbb{P}}, z, \mu^{2}\right)=\int d t f_{i}^{D, t}\left(x_{\mathbb{P}}, z, \mu^{2} ; t\right) .
$$

The resulting $\mathrm{dPDF}$ is a dimensionless quantity, which is as expected for the probability of finding a parton in a Pomeron with no constraints imposed on the value of the Pomeron momentum.

Below in Secs. III B and III C we will present and outline the derivation of hadronic cross-section integrals for the instanton production processes shown in Figs. 1(a)-2(b). Readers primarily interested in the final expressions for these cross sections can skip directly to Sec. III D where we present a brief summary of this section's results.

\section{B. Instanton production in gluon fusion processes 2 (b)}

The total cross section for the central instanton production in Fig. 2(b) is essentially a standard expression given by the convolution of the $t$-independent diffractive PDFs in (3.3) with the parton-level instanton cross section $\hat{\sigma}_{i j}$ computed using the formalism of Sec. II,

$$
\begin{aligned}
\sigma_{p p \rightarrow I}^{(2 b)}= & S^{2} \int_{\hat{s}_{\min }}^{\hat{s}_{\max }} d x_{\mathbb{P}, 1} d x_{\mathbb{P}, 2} d z_{1} d z_{2} \sum_{i j} f_{i}^{D}\left(x_{\mathbb{P}, 1}, z_{1}, Q^{2}\right) \\
& \times f_{j}^{D}\left(x_{\mathbb{P}, 2}, z_{2}, Q^{2}\right) \cdot \hat{\sigma}_{i j}\left(\hat{s}, Q^{2}, Q^{2}\right) .
\end{aligned}
$$

In this expression $S^{2}$ denotes the rapidity gap survival factor, and $\hat{\sigma}_{i j}$ is the partonic instanton cross section for the initial partons $i$ and $j$. The kinematic-invariant energy of the instanton is $\hat{s}=x_{1} x_{2} s_{\mathbb{P} p}$, where $x_{1}=x_{\mathbb{P}, 1} z_{1}$ and $x_{2}=x_{\mathbb{P}, 2} z_{2}$ are the proton momentum fractions carried by each of the two gluons entering the instanton in Fig. 2(b), and $s_{p p}$ is the center-of-mass energy squared of the hadron collider. In our setup we assume that the Pomeron momentum fractions $x_{\mathbb{P}, 1}$ and $x_{\mathbb{P}, 2}$ are measured and fixed by detecting the original protons with the momenta $p_{L}=x_{L} p=\left(1-x_{\mathbb{P}}\right) p$. The virtualities of the two single gluon states emitted from the two Pomerons are fixed in (3.4) at the same characteristic values $Q=2 \mathrm{GeV}$ as in Ref. [11].

The parton-level instanton cross section $\hat{\sigma}_{i j}\left(\hat{s}, Q^{2}, Q^{2}\right)$ that appears on the right-hand side of (3.4), and later in (3.10), is given by the formula (2.6) with the field insertion given by (2.15), as explained in Sec. II.

The limits in the integral (3.4) are written in terms of the instanton energy squared $\hat{s}_{\min }$ and $\hat{s}_{\max }$. The value of $\hat{s}_{\max }=$ $x_{\mathbb{P}, 1} x_{\mathbb{P}, 2} s_{p p}$ is fixed kinematically. Formally we have to put $\hat{s}_{\text {min }}=0$. However, small mass $\left(\sqrt{\hat{s}}=M_{\text {inst }}\right)$ instantons have a large size, $\rho$, and correspondingly we have to work with a large QCD coupling $\alpha_{s}(\mu=1 / \rho)$. Here we cannot guarantee the accuracy of our theory. Moreover, in this low $\hat{s}$ region it appears impossible to distinguish the instanton from the background and underlying event experimentally. Therefore we have to choose appropriate cuts on the final state to select the events where the role of low mass instantons is suppressed. The value of $\hat{s}_{\min }$ is chosen in such a way that after these cuts the final contribution from the $\hat{s}<\hat{s}_{\text {min }}$ region becomes negligible.

If one wishes to further suppress the low mass instantons, ${ }^{7}$ one can additionally require that the spectator jet [for example, the upper gluon in Fig. 2(a)] has a large transverse momentum, $Q_{t}$. In this case we have to work with the unintegrated parton distribution, $F_{i}^{D}\left(x_{\mathbb{P}}, z, Q_{t}^{2}\right)$, where not only the energy momentum fraction $x=x_{\mathbb{P}} z$ carried by the parton $i$ but also its transverse momentum $Q_{t}$ are fixed. This is used to determine the differential cross section with respect to the $Q_{t}$ of the spectator jet, which reads

$$
\begin{aligned}
Q_{t}^{2} \frac{d}{d Q_{t}^{2}} \sigma_{p p \rightarrow I+i}^{(2 b)}= & S^{2} \int \frac{d x_{\mathbb{P}, 1} d x_{\mathbb{P}, 2}}{x_{\mathbb{P}, 1}} \frac{d z_{1}}{z_{1}} d z_{2} \\
& \times \sum_{j} F_{i}^{D}\left(x_{\mathbb{P}, 1}, z_{1}, Q_{t}^{2}\right) f_{j}^{D}\left(x_{\mathbb{P}, 2}, z_{2}, Q^{2}\right) \\
& \cdot \hat{\sigma}_{i j}\left(\hat{s}, Q_{t}^{2}, Q^{2}\right) .
\end{aligned}
$$

Note that the virtuality of the first gluon $Q_{t}$ is set by the transverse momentum of the spectator jet and is different from the intrinsic virtuality of the second gluon that we keep at $Q=2 \mathrm{GeV}$ as before.

In general, an unintegrated distribution, $F\left(x, Q_{t}^{2}, \mu^{2}\right)$, at leading order can be calculated from the usual integrated parton distribution using the DGLAP evolution equation, as explained in $[25],{ }^{8}$

$$
\begin{aligned}
F_{i}\left(x, Q_{t}^{2}, \mu^{2}\right)= & T_{i}\left(Q_{t}, \mu\right) \frac{\alpha_{s}\left(Q_{t}\right)}{2 \pi} \\
& \times \sum_{j} \int_{x}^{1-\Delta\left(Q_{t}\right)} P_{i j}(\tilde{z}) a_{j}\left(\frac{x}{\tilde{z}}, Q_{t}\right) d \tilde{z} .
\end{aligned}
$$

Here $P_{i j}(\tilde{z})$ is the real part of the splitting functions, $\Delta\left(Q_{t}\right)=Q_{t} /\left(Q_{t}+\mu\right)$ provides the angular ordering of a subsequent gluon emission, and $a_{i}\left(x, \mu^{\prime}\right)$ denotes the integrated distributions taken at the scale $\mu^{\prime}=Q_{t}$.

\footnotetext{
${ }^{7}$ And consequentially pay the price of having much lower instanton cross sections (as was also discussed in Sec. 3 of Ref. [9]).

${ }^{8}$ One could also use other formalisms (such as the transverse momentum dependent formalism [26] or other unintegrated PDF prescriptions) for the calculation of the unintegrated PDFs but we would expect the difference between these approaches to be a next-to-leading-order effect. The approach chosen here is quite standard in the literature and has been used in, e.g., [27,28] to successfully describe experimental data.
} 
Specifically, $a_{i}\left(x, \mu^{\prime}\right)=x g\left(x, \mu^{\prime}\right)$ for $i=g$ and $a_{i}\left(x, \mu^{\prime}\right)=$ $x q_{f}\left(x, \mu^{\prime}\right)$ for $i=q_{f}$.

The Sudakov factor $T\left(Q_{t}, \bar{\mu}\right)$ on the right-hand side of (3.6) represents the probability not to emit additional partons with transverse momentum $k_{t}>Q_{t}$ which would change the final virtuality and the transverse momentum. At leading order (LO) these additional partons can be radiated within the interval $Q_{t}<k_{t}<\bar{\mu}$. The scale $\bar{\mu}$ is set by the instanton mass $\bar{\mu} \simeq M_{\text {Inst }}$ (see [29]),

$T_{i}\left(Q_{t}, \bar{\mu}\right)=\exp \left(-\int_{Q_{t}^{2}}^{\bar{\mu}^{2}} \frac{d k^{2}}{k^{2}} \frac{\alpha_{s}\left(k^{2}\right)}{2 \pi} \sum_{j} \int_{0}^{1-\Delta(k)} P_{j i}(\tilde{z}) d \tilde{z}\right)$,

where for the $g \rightarrow g g$ splitting we have to insert a factor $z$ in front of $P_{g g}(z)$ to account for the identity of the produced gluons; $\Delta(k)=k /(k+\bar{\mu})$. When the value of $Q_{t}>\bar{\mu}$, there is no phase space for LO gluon emissions, and this implies that the Sudakov factor becomes $T_{i}\left(Q_{t}, \bar{\mu}\right)=1$. With this simplification, the unintegrated distribution can be written as [30]

$$
F_{i}\left(x, Q_{t}^{2}\right)=\frac{d a_{i}\left(x, Q_{t}\right)}{d \ln Q_{t}^{2}} .
$$

Diffractive events with LRGs are then described by the unintegrated diffractive distribution $F_{i}^{D}$ given by the same expression (3.8) with the incoming distribution $a_{i}$ replaced by $x f_{i}^{D}\left(x_{\mathbb{P}}, z, \mu^{\prime}\right)$,

$$
F_{i}^{D}\left(x_{\mathbb{P}}, z, Q_{t}^{2}\right)=\frac{d}{d \ln Q_{t}^{2}} x_{\mathbb{P}} z f_{i}^{D}\left(x_{\mathbb{P}}, z, \mu^{2}=Q_{t}^{2}\right) .
$$

We can now use the formula (3.5) to write down the total cross section for the process in Fig. 2(b) in terms of unintegrated distributions,

$$
\begin{aligned}
\sigma^{(2 b)}= & S^{2} \int \frac{d x_{\mathbb{P}, 1} d x_{\mathbb{P}, 2}}{x_{\mathbb{P}, 1} x_{\mathbb{P}, 2}} \frac{d z_{1} d z_{2}}{z_{1} z_{2}} \int \frac{d Q_{t, 1}^{2} d Q_{t, 2}^{2}}{Q_{t, 1}^{2} Q_{t, 2}^{2}} \\
& \times \sum_{i j} F_{i}^{D}\left(x_{\mathbb{P}, 1}, z_{1}, Q_{t, 1}^{2}\right) F_{j}^{D}\left(x_{\mathbb{P}, 2}, z_{2}, Q_{t, 2}^{2}\right) \\
& \times \hat{\sigma}_{i j}\left(\hat{s}, Q_{t, 1}^{2}, Q_{t, 2}^{2}\right) .
\end{aligned}
$$

The factors of $1 / x_{\mathbb{P}}$ in (3.10), and before that in (3.5), are the consequence of the factor of $x_{\mathbb{P}}$ included in the definition of $F_{i}^{D}$ thanks to $a_{i}=x f_{i}^{D}=x_{\mathbb{P}} z f^{D}\left(x_{\mathbb{P}}, z, \ldots\right)$ in (3.9), (3.8), and (3.6). ${ }^{9}$

Similar to the earlier case in (3.4), the parton-level instanton cross section $\hat{\sigma}_{i j}\left(\hat{s}, Q_{t, 1}^{2}, Q_{t, 2}^{2}\right)$ that appears on

\footnotetext{
${ }^{9}$ It is convenient since in this form at a very small $x_{\mathbb{P}}$ (and/or $\left.z\right)$ we deal only with a weak (mainly logarithmic) $x_{\mathbb{P}}$ (or $z$ ) dependence.
}

the right-hand side of (3.10) is found by plugging in the field insertion given by (2.16) into the integral (2.6). Note that the transverse momentum $Q_{t}^{2}$ is now an integration variable in (3.10).

The expression (3.10) is relevant to the case where we choose to trigger on a spectator jet with the transverse momentum $Q_{t}$ in a certain range. Otherwise, one should use the simpler expression in (3.4).

\section{Instanton production processes 1(a), 1(b), and 2(a)}

Cross-section formulas for the remaining central instanton production processes in Figs. 1(a),1(b), and 2(a) require a little more work. In all these cases, as can be seen in the figures, there is a pair of partons coming from a Pomeron to the instanton that forms a loop and involves an integration over (the longitudinal and transverse components of) the loop momenta. Also the parton-level instanton amplitudes can now contain 2, 3, or 4 initial partons.

Selecting one pair of gluons entering the instanton vertex from a Pomeron [either one of the two $(g g)$ pairs in Fig. 1(a) or the (gg) pair in Fig. 2(a)] we can write the loop integral over the transverse momentum components $Q_{t}$ as

$$
\begin{gathered}
\int \frac{d Q_{t}^{2}}{Q_{t}^{2}} \hat{F}_{g}\left(x_{\mathbb{P}}, z, Q_{t}^{2} ; t\right) \mathcal{A}_{(g g) \rightarrow \text { Inst }} \\
\propto \int \frac{d Q_{t}^{2}}{Q_{t}^{2}} \hat{F}_{g}\left(x, Q_{t}^{2} ; t\right) e^{-2 Q_{t} \rho} .
\end{gathered}
$$

The quantity $\mathcal{A}_{(g g) \rightarrow \text { Inst }}$ on the left-hand side of (3.11) denotes the instanton amplitude with the initial state that includes the gluon pair $(g g)$ which appears in the loop integral. This integral is convergent at large $Q_{t}^{2}$ thanks to the virtual gluons in the $(g g)$ state that lead to the appearance of the form factor $J^{2}\left(Q_{t} \rho\right) \propto e^{-2 Q_{t} \rho}$ on the right-hand side of (3.11) [as dictated by (2.17) and (2.14)]. The convergence at small $Q_{t} \rightarrow 0$ is provided by the $T\left(Q_{t}, \mu\right)$ factor hidden in $\hat{F}_{g}$.

We also note that the PDF function $\hat{F}_{g}$ in (3.11) is not the diffractive PDF $F_{g}^{D}$, but the gluon distribution $F_{i=g}$ calculated via (3.6) based on the standard inclusive distribution $a_{i}=x f_{i}(x, \mu, t)$ at fixed $t .{ }^{10}$ That is, the Pomeron exchange is represented by the singlet (in color and in flavor) parton distribution $f_{i}(x, \mu, t)$.

We also note that for the parton distribution $\hat{F}$ appearing in (3.11) one should use the generalized (or skewed) distribution since the longitudinal momentum fractions, $x^{\prime}=x_{\mathbb{P}}(1-z)$ and $x=x_{\mathbb{P}} z$, transferred through the left

\footnotetext{
${ }^{10}$ Indeed, the standard PDF describes the probability to find the corresponding parton. Our case concerns the probability amplitude that is given by the generalized parton distribution (GPD). The amplitude described by the GPD, by definition, provides the interaction across the rapidity gap.
} 
and the right gluons in the loop are different. Nevertheless, for $x_{\mathbb{P}} \ll 1$ this generalized parton distribution function, $\operatorname{GPD}(X, \xi, \mu)$, can be calculated from the usual, measured in deep inelastic scattering, $\operatorname{PDF}(x, \mu)$ with the help of a Shuvaev transform [31,32] with $O(\xi)$ accuracy. Here we use the conventional for GPD functions notations:

$x=\xi+X=z x_{\mathbb{P}}, \quad x^{\prime}=\xi-X=(1-z) x_{\mathbb{P}}$,

so that $\xi=x_{\mathbb{P}} / 2$ and $X=(z-1 / 2) x_{\mathbb{P}}$. In this notation the contribution to the loop integral from integrating over $z$ takes the form ${ }^{11}$

$$
-\frac{i}{\pi} \int_{-1}^{1} \frac{\xi d X}{(X-\xi+i \epsilon)(X+\xi-i \epsilon)} \operatorname{GPD}(X, \xi) .
$$

Since the $\operatorname{GPD}(X, \xi)$ distribution decreases for $|X| \gg \xi$, the integral can be calculated by closing the contour on the $1 /(X-\xi)$ pole which sets $z=1$.

We can now express the cross section for the process shown in Fig. 1(a) in the form

$$
\begin{aligned}
\sigma^{(1 a)}= & S^{2} \frac{1}{(4 \pi)^{4}} \int_{\hat{s}_{\min }}^{\hat{s}_{\max }} \frac{d x_{1} d x_{2}}{x_{1} x_{2}} \int d t_{1} d t_{2} \\
& \times \int \frac{d Q_{1 t}^{2}}{Q_{1 t}^{2}} R_{1 g} F_{1 g}\left(x_{1}, Q_{1 t}^{2} ; t_{1}\right) \\
& \times \int \frac{d \bar{Q}_{1 t}^{2}}{\bar{Q}_{1 t}^{2}} R_{1 g} F_{1 g}\left(x_{1}, \bar{Q}_{1 t}^{2} ; t_{1}\right) \\
& \times \int \frac{d Q_{2 t}^{2}}{Q_{2 t}^{2}} R_{2 g} F_{2 g}\left(x_{2}, Q_{2 t}^{2} ; t_{2}\right) \\
& \times \int \frac{d \bar{Q}_{2 t}^{2}}{\bar{Q}_{2 t}^{2}} R_{2 g} F_{2 g}\left(x_{2}, \bar{Q}_{2 t}^{2} ; t_{2}\right) \\
& \times \hat{\sigma}_{(g g)+(g g) \rightarrow \operatorname{Inst}}\left(\hat{s}, Q_{1 t}^{2}, \bar{Q}_{1 t}, Q_{2 t}^{2}, \bar{Q}_{2 t}\right) .
\end{aligned}
$$

Here, as before, $S^{2}$ denotes the gap survival factor. The factors $R_{1 g}\left(R_{2 g}\right)$ are the ratios of the generalized parton distributions to the nonskewed PDFs, $R_{i}=$ $\operatorname{GPD}_{i}(\xi, \xi ; \mu) / f_{i}(2 \xi, \mu)$ [31], where we set $X=\xi$ (i.e., $z=1)$, and the elementary instanton cross section $\hat{\sigma}_{(g g)+(g g) \rightarrow \text { Inst }}$ is also calculated at $z_{1}=1=z_{2}$. The unintegrated distributions $F_{i g}$ are calculated from the standard gluon PDFs via (3.6).

The parton-level instanton cross section $\hat{\sigma}_{(g g)+(g g) \rightarrow \text { Inst }}(\hat{s}$, $\left.Q_{1 t}^{2}, \bar{Q}_{1 t}, Q_{2 t}^{2}, \bar{Q}_{2 t}\right)$ is given by the integral (2.6) with the field insertion given by Eqs. (2.28) and (2.21) derived in Sec. II.

\footnotetext{
${ }^{11}$ Since $x_{\mathbb{P}}$ is measured and fixed, the integration over $z$ translates into the integration over $X$. The denominator $1 /((X-$ $\xi)(X+\xi))$ comes from the gluon polarization vectors which, using gauge invariance, can be written in the form $\epsilon_{\mu}=-Q_{t, \mu} / x$.
}

For each of the two gluon loops in Fig. 1(a) the expression in (3.14) contains the factor $\frac{1}{(4 \pi)^{2}} \int \frac{d Q_{i t}^{2}}{Q_{i t}^{2}} \times$ $R_{i g} F_{i g}\left(x_{i}, Q_{i t}^{2} ; t_{i}\right) \int \frac{d \bar{Q}_{i t}^{2}}{\bar{Q}_{i t}^{2}} R_{i g} F_{i g}\left(x_{i}, \bar{Q}_{i t}^{2} ; t_{i}\right) \quad$ reflecting the fact that the gluon loop contribution to the amplitude is squared in the cross section. Note that writing the cross section in terms of the unintegrated distributions in (3.14) we, to a large extent, solve the problem of the choice of an appropriate factorization scale. The characteristic values of $Q_{i t}$ in the integrals (and correspondingly the factorization scale) are determined by the factors $\exp \left(-2\left(Q_{i t} \rho+\bar{Q}_{i t} \bar{\rho}\right)\right)$ already present in the instanton cross section $\hat{\sigma}_{(g g)+(g g) \rightarrow \operatorname{Inst}}\left(\hat{s}, Q_{1 t}^{2}, \bar{Q}_{1 t}, Q_{2 t}^{2}, \bar{Q}_{2 t}\right)$ and in the Sudakov factors $T\left(Q_{i t}, M_{\text {inst }}\right)$ hidden in the unintegrated distributions $F\left(x_{i}, Q_{i t}^{2} ; t_{i}\right)$. The first factor prefers lower values of $Q_{i t}$ (for a fixed instanton size) while the $T$ factor prefers higher values of the transverse momentum as $T$ increases with $Q_{i t}$. At the same time the Sudakov $T$ factor ensures the infrared convergence of the $Q_{i t}$ integrals in (3.14).

Calculating the $p+p \rightarrow p+$ Instanton $+p$ cross section we have also included into (3.14) integration over the transverse momenta, $p_{t}$, of outgoing protons which amounts to the $d t_{1} d t_{2}$ integral in (3.14). ${ }^{12}$ The $p_{t}$ dependence is hidden in the generalized parton distribution, $F_{g}\left(x, Q_{t} ; t\right)$, where $t$ is the momentum transfer squared $t_{1}=-p_{t 1}^{2} \quad\left(t_{2}=-p_{t 2}^{2}\right)$. This $t$ dependence originates mainly from the proton form factors in (3.2). In our calculations we will take the exponential parametrization,

$$
\frac{d \sigma}{d t_{1} d t_{2}} \propto e^{b\left(t_{1}+t_{2}\right)}
$$

with the slope $b=6 \mathrm{GeV}^{-2}$. This value of $b$ is consistent with that used in the $\mathrm{H} 1$ analysis [23]. [In terms of (3.2) the slope is given by $b=B_{\mathbb{P}}-2 \alpha_{\mathbb{P}}^{\prime}(0) \log x_{\mathbb{P}}$.]

Within the DGLAP approach the "Pomeron-partonparton" vertex conserves parton helicity. On the other hand, fermion zero modes of light quarks in the instanton background, which at the leading order describe the quark-antiquark-instanton interaction, contain quarks of different helicities (such as $q_{L} \bar{q}_{R} \rightarrow$ Instanton). Therefore in (3.14), (3.16), (3.17), and (4.14) we have set the initial partons to be gluons and neglected the quark loops.

The cross section for the process shown in Fig. 1(b) can be written analogously to (3.14) as follows:

\footnotetext{
${ }^{12}$ The integration over the longitudinal momentum fractions is written in (3.14) as the integral $\int d x_{1} d x_{2}$. Note that in the earlier equations (3.4) and (3.10), the corresponding $d t$ integrations were not displayed as they were already included in the Pomeron flux in accordance with (3.3).
} 


$$
\begin{aligned}
\sigma^{(1 b)}= & S^{2} \int_{\hat{s}_{\min }}^{\hat{s}_{\max }} \frac{d x_{1} d x_{2}}{x_{1} x_{2}} \int d t_{1} d t_{2} \\
& \times\left[\int \frac{d Q_{t}^{2}}{Q_{t}^{4}} R_{1 g} F_{1 g}\left(x, Q_{t}^{2} ; t_{1}\right) R_{2 g} F_{2 g}\left(x_{2}, Q_{t}^{2} ; t_{2}\right)\right]^{2} \\
& \times \frac{\pi^{2}}{\left(N_{c}^{2}-1\right)^{2}} \hat{\sigma}_{g g \rightarrow \text { Inst }}\left(\hat{s}, Q_{t}^{2}, Q_{t}^{2}\right)
\end{aligned}
$$

In (3.16) (and also in subsequent expressions below) we use the shorthand notation $\left[\int \frac{d Q_{t}^{2}}{Q_{t}^{4}} \cdots\right]^{2}=\int \frac{d Q_{t}^{2}}{Q_{t}^{4}} \cdots \int \frac{d \bar{Q}_{t}^{2}}{\bar{Q}_{t}^{4}} \cdots$ and $\hat{\sigma}_{g g \rightarrow \text { Inst }}\left(\hat{s}, Q_{t}^{2}, \bar{Q}_{t}^{2}, Q_{t}^{2}, \bar{Q}_{t}^{2}\right)=\hat{\sigma}_{g g \rightarrow \text { Inst }}\left(\hat{s}, Q_{t}^{2}, Q_{t}^{2}\right)$. The parton-level instanton cross section is computed using the field insertion $\langle\cdots\rangle_{(g+g)_{Q_{t}, \bar{Q}_{t}}}$ defined in (2.16).

The factor $1 /\left(N_{c}^{2}-1\right)$ in (3.16) reflects the requirement that the partons in the upper and lower parts of Fig. 1(b) must have the same color. This expression (3.16) has the same form as that given by Eq. (7) in Ref. [12]. ${ }^{13}$

Strictly speaking, we have to sum the processes in Figs. 1 (a) and 1(b) at the level of amplitudes. However, since the Pomeron intercept $\alpha_{\mathbb{P}}(0)$ is close to 1, the amplitude Fig. 1 (a) is (mainly) real while the amplitude Fig. 1(b) is (mainly) imaginary. Thus we may neglect their interference and sum over the respective cross sections.

Finally, we can now present the expression for the cross section of the process in Fig. 2(a), again using the shorthand notation in terms of the squared $d Q_{2 t}^{2}$ integral,

$$
\begin{aligned}
\sigma^{(2 a)}= & \frac{S^{2}}{(4 \pi)^{2}} \int_{\hat{s}_{\min }}^{\hat{s}_{\max }} \frac{d x_{\mathbb{P}, 1} d x_{2}}{x_{\mathbb{P}, 1} x_{2}} \frac{d z_{1}}{z_{1}} \\
& \times \sum_{i} \int \frac{d Q_{1 t}^{2}}{Q_{1 t}^{2}} F_{1 i}^{D}\left(x_{\mathbb{P}, 1}, z_{1}, Q_{1 t}\right) \\
& \times \int d t_{2}\left[\int \frac{d Q_{2 t}^{2}}{Q_{2 t}^{2}} R_{2 g} F_{2 g}\left(x_{2}, Q_{2 t} ; t_{2}\right)\right]^{2} \\
& \times \hat{\sigma}_{i+(g g) \rightarrow \text { Inst }}\left(\hat{s}, Q_{1 t}^{2}, Q_{2 t}^{2}\right) .
\end{aligned}
$$

As before, the parton-level instanton cross sections $\hat{\sigma}_{g g \rightarrow \text { Inst }}$ and $\hat{\sigma}_{i+(g g) \rightarrow \text { Inst }}$ appearing in the hadronic cross-section formulas (3.16) and (3.17) are given by the integral (2.6) over the instanton collective coordinates, and the relevant field insertion was derived in (2.23).

Comparing the expressions for the cross sections of the processes in Figs. 1(a),2(a), and 2(b) in Eqs. (3.14), (3.17), and (3.10), we see that when a Pomeron interacts with the instanton via a gluon loop, the cross-section integral receives a factor of

\footnotetext{
${ }^{13}$ Note also that the momentum fractions $z$ and $z^{\prime}$ in Fig. 1(b) correspond to the different components of the 4-vector momentum. In the upper part of the diagram the proton momentum $p_{\mu}$ is mainly $p^{+}$and $z$ corresponds to the fraction of $p^{+}$momentum while in the lower part of the diagram we deal with the $p^{-}$ component. For the left gluon both the $p^{+}$and $p^{-}$components are very small.
}

$$
\frac{1}{(4 \pi)^{2}} \int d t\left[\int \frac{d Q_{t}^{2}}{Q_{t}^{2}} R_{g} F_{g}\left(x, Q_{t} ; t\right)\right]^{2}
$$

from the loop integral squared. On the other hand, for a single parton exchange between the Pomeron and the instanton, with the second parton being a spectator jet, the cross section gets a factor of

$$
\int \frac{d Q_{t}^{2}}{Q_{t}^{2}} F_{i}^{D}\left(x_{1}, Q_{t}\right)
$$

Notice that (3.19) does not depend on the $t$ variable, and we can also simplify (3.18) by carrying out the $d t$ integration with the help of the cross-section parametrization formula (3.15); that is, we can replace (3.18) by

$$
\frac{1}{b} \frac{1}{(4 \pi)^{2}}\left[\int \frac{d Q_{t}^{2}}{Q_{t}^{2}} R_{g} F_{g}\left(x, Q_{t}\right)\right]^{2}
$$

As a result, both final expressions in the factors (3.19) and (3.20) contain no integrations over $t$ left over.

\section{Summary of PDFs and cross-section formulas}

In our discussion of hadronic cross sections in the preceding section we have encountered the following diffractive parton distribution functions:

$$
\begin{array}{r}
\mathrm{dPDF} \text { at fixed } t: f_{i}^{D, t}\left(x_{\mathbb{P}}, z, \mu^{2} ; t\right) \\
=f_{\mathbb{P} / p}\left(x_{\mathbb{P}}, t\right) f_{i}\left(z=\frac{x}{x_{\mathbb{P}}}, \mu^{2}\right) ; \\
\text { Pomeron flux }: f_{\mathbb{P} / p}\left(x_{\mathbb{P}}, t\right)=g_{N}^{2} \frac{e^{B_{\mathbb{P}} t}}{x_{\mathbb{P}}^{2 \alpha_{\mathbb{P}}(t)-1}} ;
\end{array}
$$

PDF inside the Pomeron: $f_{i}(z, \mu)$;

$$
\mathrm{dPDF}: f_{i}^{D}\left(x_{\mathbb{P}}, z, \mu^{2}\right)=\int d t f_{i}^{D, t}\left(x_{\mathbb{P}}, z, \mu^{2} ; t\right)
$$

as well as the so-called unintegrated diffractive distribution $F_{i}^{D}\left(x_{\mathbb{P}}, z, Q_{t}^{2}, \mu^{2}\right)$ that we will present in a moment.

The most elementary object in this list is the diffractive PDF at fixed $t$ given in (3.21), where $t$ is defined as the momentum transfer squared through the Pomeron and $\mu^{2}$ is the factorization scale. The distribution function $f_{i}^{D, t}\left(x_{\mathbb{P}}, z, \mu^{2} ; t\right)$ is the probability to find a parton $i$ in the Pomeron where the parton carries a fraction $z$ of the Pomeron momentum and the Pomeron carries a fraction $x_{\mathbb{P}}$ of the proton momentum. The Pomeron is assumed to have a fixed value of momentum squared $t$, and as a result the $\mathrm{dPDF}$ in (3.21) has mass dimension -2 , 


$$
\begin{gathered}
{\left[f_{i}^{D, t}\left(x_{\mathbb{P}}, z, \mu^{2} ; t\right)\right]=\left[f_{\mathbb{P} / p}\left(x_{\mathbb{P}}, t\right)\right]=\left[g_{N}^{2}\right]=-2,} \\
{\left[f_{i}(z, \mu)\right]=0 .}
\end{gathered}
$$

The Pomeron flux function $f_{\mathbb{P} / p}\left(x_{\mathbb{P}}, t\right)$ in (3.22) and the $i$ th parton PDF inside the Pomeron $f_{i}(z, \mu)$ in (3.23) are used as the building blocks to construct the dPDF at fixed $t$ in Eq. (3.21). After integrating over $d t$ we obtain the more conventionally normalized probability - the $t$-independent diffractive PDF defined in (3.24) — which is a dimensionless quantity.

It is the $t$-independent $\mathrm{dPDF}$ (3.24) that is relevant for the central instanton production process in Fig. 2(b). The total $p p$ cross-section formula for the process 2(b) when we do not tag the spectator jets (i.e., no requirements are placed on their transverse momentum) is given by

$$
\begin{aligned}
\sigma^{(2 b)}= & S^{2} \int_{\hat{s}_{\min }}^{\hat{s}_{\max }} d x_{\mathbb{P}, 1} d x_{\mathbb{P}, 2} d z_{1} d z_{2} \\
& \times \sum_{i j} f_{i}^{D}\left(x_{\mathbb{P}, 1}, z_{1}, Q_{1}^{2}\right) f_{j}^{D}\left(x_{\mathbb{P}, 2}, z_{2}, Q_{2}^{2}\right) \\
& \cdot \hat{\sigma}_{i j}\left(\hat{s}, Q_{1}^{2}, Q_{2}^{2}\right) .
\end{aligned}
$$

If we choose to trigger on a spectator jet with transverse momentum $Q_{t}$ in a certain range, the cross-section integral for $\sigma^{(2 b)}$ takes the form

$$
\begin{aligned}
\sigma^{(2 b)}= & S^{2} \int \frac{d x_{\mathbb{P}, 1} d x_{\mathbb{P}, 2} d z_{1} d z_{2}}{x_{\mathbb{P}, 1} x_{\mathbb{P}, 2} z_{1} z_{2}} \\
& \times \sum_{i j} \int \frac{d Q_{t, 1}^{2} d Q_{t, 2}^{2}}{Q_{t, 1}^{2} Q_{t, 2}^{2}} F_{i}^{D}\left(x_{\mathbb{P}, 1}, z_{1}, Q_{t, 1}\right) \\
& \cdot F_{j}^{D}\left(x_{\mathbb{P}, 2}, z_{2}, Q_{t, 2}^{2}\right) \hat{\sigma}_{i j}\left(\hat{s}, Q_{t, 1}^{2}, Q_{t, 2}^{2}\right),
\end{aligned}
$$

which now involves the new unintegrated diffractive PDF function, $F_{i}^{D}\left(x_{\mathbb{P}}, z, Q_{t}^{2}\right)$ taken at the scale $\mu^{2}=\hat{s}$. For a transverse momentum, $Q_{t}$, greater than the instanton mass the expression for the unintegrated dPDF can be written as a derivative of the original $\mathrm{dPDF} f_{i}^{D}$,

$$
\text { For } \begin{aligned}
Q_{t} & >M_{\text {Inst }}: F_{i}^{D}\left(x_{\mathbb{P}}, z, Q_{t}^{2}\right) \\
& =\frac{d}{d \log Q_{t}^{2}} x_{\mathbb{P}} z f_{i}^{D}\left(x_{\mathbb{P}}, z, \mu^{2}\right) .
\end{aligned}
$$

For lower values of $Q_{t}$, one should instead use the more general expression, analogous to (3.6), that involves the Sudakov form factor $T_{i}\left(Q_{t}, \mu\right)$ where we set the scale $\mu$ to be equal to the instanton mass $M_{\text {inst }}=\sqrt{\hat{s}}$.

It is easy to see that both expressions, (3.26) and (3.27), have the correct dimension for the cross section since the unintegrated $\mathrm{dPDF}$ in (3.28) and the $t$-independent $\mathrm{dPDF}$ function $f_{i}^{D}\left(x_{\mathbb{P}}, z, \mu^{2}\right)$ from which it is derived are both dimensionless.
For the cross section for the process in Fig. 1(a) we have

$$
\begin{aligned}
\sigma^{(1 a)}= & S^{2} \int_{\hat{s}_{\min }}^{\hat{s}_{\max }} \frac{d x_{1} d x_{2}}{x_{1} x_{2}} \int d t_{1} d t_{2} \frac{1}{(4 \pi)^{4}} \\
& \times\left[\int \frac{d Q_{1 t}^{2}}{Q_{1 t}^{2}} R_{1 g} F_{1 g}\left(x_{1}, Q_{1 t}^{2} ; t_{1}\right)\right]^{2} \\
& \times\left[\int \frac{d Q_{2 t}^{2}}{Q_{2 t}^{2}} R_{2 g} F_{2 g}\left(x_{2}, Q_{2 t}^{2} ; t_{2}\right)\right]^{2} \\
& \times \hat{\sigma}_{(g g)+(g g) \rightarrow \text { Inst }}\left(\hat{s}, Q_{1 t}^{2}, Q_{2 t}^{2}\right) .
\end{aligned}
$$

Here the functions $F_{g}\left(x, Q_{t}^{2} ; t\right)$ denote standard (i.e., nondiffractive) unintegrated gluon PDFs at fixed $t$ (and with the scale chosen at $\mu^{2}=Q_{t}^{2}$ ). The factors $R_{g}$ are the known ratios of the generalized (skewed) PDFs to the standard ones given by $F_{g}$.

How do we carry out the integrations in (3.29) over $d t_{1} d t_{2}$ ? The $t$ dependence of the cross section is hidden in the unintegrated PDFs $F_{g}\left(x, Q_{t}^{2} ; t\right)$ and can be traced to the proton form factors contained therein. Adopting a simple exponential parametrization of the cross section,

$$
\frac{d \sigma}{d t_{1} d t_{2}} \propto e^{b\left(t_{1}+t_{2}\right)}
$$

with the slope $b=6 \mathrm{GeV}^{-2}$, we can effectively carry out the integrations over $d t_{1} d t_{2}$ in (3.29) with the result

$$
\begin{aligned}
\sigma^{(1 a)}= & \frac{1}{b^{2}} \frac{S^{2}}{(4 \pi)^{4}} \int_{\hat{s}_{\min }}^{\hat{s}_{\max }} \frac{d x_{1} d x_{2}}{x_{1} x_{2}}\left[\int \frac{d Q_{1 t}^{2}}{Q_{1 t}^{2}} R_{1 g} F_{1 g}\left(x_{1}, Q_{1 t}^{2}\right)\right]^{2} \\
& \times\left[\int \frac{d Q_{2 t}^{2}}{Q_{2 t}^{2}} R_{2 g} F_{2 g}\left(x_{2}, Q_{2 t}^{2}\right)\right]^{2} \\
& \times \hat{\sigma}_{(g g)+(g g) \rightarrow \text { Inst }}\left(\hat{s}, Q_{1 t}^{2}, Q_{2 t}^{2}\right) .
\end{aligned}
$$

There is now a factor of $b^{-2}$ in front of the integral and the unintegrated gluon PDFs $F_{g}\left(x, Q_{t}^{2}\right)$ have now lost their $t$ dependence. In the simplified case, where one can ignore the Sudakov form factor, they are given by the analogous to (3.28) expression,

Unintegrated PDF: $F_{g}\left(x, Q_{t}^{2}\right)=\frac{d}{d \log Q_{t}^{2}} x g\left(x, Q_{t}^{2}\right)$,

where $g\left(x, \mu^{2}\right)=f_{g}\left(x, \mu^{2}\right)$ is the usual gluon PDF.

The expression for $F_{g}\left(x, Q_{t}^{2}\right)$ is dimensionless, and taking into account the mass-dimension 4 factor of $1 / b^{2}$ along with the fact that the parton-level instanton cross section $\hat{\sigma}_{(g g)+(g g) \rightarrow \text { Inst }}$ has mass-dimension equal to -6 on account of having four initial state partons rather than the 
usual two, we thus obtain the correct dimensionality -2 of the total cross section in (3.32). ${ }^{14}$

In the case of the low spectator jet transverse momentum $Q_{t}<\mu=M_{\text {inst }}$ where the Sudakov form factor cannot be ignored, the unintegrated PDF (for parton $i$ ) is given by the more general expression (3.6).

For the remaining processes, there are no new ingredients with the relevant PDFs being already defined above, and from (3.16) and (3.17) we have

$$
\begin{aligned}
\sigma^{(1 b)}= & \frac{1}{b^{2}} S^{2} \int_{\hat{s}_{\min }}^{\hat{s}_{\max }} \frac{d x_{1} d x_{2}}{x_{1} x_{2}} \\
& \times\left[\int \frac{d Q_{t}^{2}}{Q_{t}^{4}} R_{1 g} F_{1 g}\left(x, Q_{1 t}^{2}\right) R_{2 g} F_{2 g}\left(x_{2}, Q_{2 t}^{2}\right)\right]^{2} \\
& \times \frac{\pi^{2}}{\left(N_{c}^{2}-1\right)^{2}} \hat{\sigma}_{g g \rightarrow \text { Inst }}\left(\hat{s}, Q_{1 t}^{2}, Q_{2 t}^{2}\right)
\end{aligned}
$$

and

$$
\begin{aligned}
\sigma^{(2 a)}= & \frac{1}{b} \frac{S^{2}}{(4 \pi)^{2}} \int_{\hat{s}_{\min }}^{\hat{s}_{\max }} \frac{d x_{\mathbb{P}, 1} d z_{1} d x_{2}}{x_{\mathbb{P}, 1} z_{1} x_{2}} \\
& \times \sum_{i} \int \frac{d Q_{1 t}^{2}}{Q_{1 t}^{2}} F_{1 i}^{D}\left(x_{\mathbb{P}, 1}, z_{1}, Q_{1 t}^{2}\right) \\
& \times\left[\int \frac{d Q_{2 t}^{2}}{Q_{2 t}^{2}} R_{2 g} F_{2 g}\left(x_{2}, Q_{2 t}^{2}\right)\right]^{2} \\
& \times \hat{\sigma}_{i+(g g) \rightarrow \mathrm{Inst}}\left(\hat{s}, Q_{1 t}^{2}, Q_{2 t}^{2}\right) .
\end{aligned}
$$

\section{PHOTON-INDUCED CENTRAL INSTANTON PRODUCTION}

In addition to Pomerons, processes with large rapidity gaps can also be initiated by a photon exchange in the $t$-channel, as shown in Figs. 3(a)-3(c). These processes include quarks and antiquarks in the initial states entering the instanton vertex.

\section{A. Fermion line insertions}

To account for processes with quarks and antiquarks on the incoming lines in the instanton process we need to specify the fermion field insertions analogously to what has been done in Sec. II for gluons. The fermion field component of the instanton solution is conventionally described by the fermion zero mode configuration [2],

$$
\hat{\psi}_{\dot{\alpha} \alpha}^{(0)}(x)=\frac{\rho}{\pi} \frac{x_{\mu} \bar{\sigma}_{\mu \dot{\alpha} \alpha}}{\left(x^{2}\right)^{1 / 2}\left(x^{2}+\rho^{2}\right)^{3 / 2}} \theta .
$$

\footnotetext{
${ }^{14}$ While it is not at all surprising that the $t$-independent gluon distribution in (3.32) was dimensionless, we should note that the $t$-dependent unintegrated PDF $F_{g}\left(x, Q_{t}^{2} ; t\right)$ appearing in (3.29) was dimensionless as well. This is different from the diffractive PDF at fixed $t$ in (3.21).
}

These fermion zero mode configurations describe both the right-handed quarks $q_{R}$ and the left-handed antiquarks $\bar{q}_{L}$ in the instanton background. Here for compactness of notation we position the instanton at $x_{0}=0$ and assume the trivial instanton orientation matrix in color space, placing the instanton in the upper $2 \times 2$ corner of the $S U$ (3) color matrix. The indices $\alpha=1,2$ and $\dot{\alpha}=1,2$ refer to the Weyl spinor components and the orientation in the $2 \times 2$ corner of the color space, respectively. The variable $\theta$ defines the Grassmann collective coordinate of the fermion zero mode and, as can easily be seen from dimensional counting, it has mass-dimension $-1 / 2$. The expression (4.1) is conventionally referred to as the normalized fermion zero mode since $\int d^{2} \theta \int d^{4} x \hat{\psi}^{(0) \dagger} \hat{\psi}^{(0)}=1$.

It is actually more convenient to switch to the dimensionless Grassmann variable $\tilde{\theta}=\theta / \rho^{1 / 2}$, in which case all grassmanian integrations in the collective coordinate measure of the instanton path integrals can be straightforwardly carried out, adding no extra dimensional factors to the instanton density $D(\rho)$ [and $D(\bar{\rho})$ ] in (2.6). After stripping off the $\tilde{\theta}$ factor we get the fermion zero mode expression with the factor $\rho^{3 / 2}$, i.e., in the form (cf. $[20,21]$ )

$$
\psi_{\dot{\alpha} \alpha}^{(0)}(x)=\frac{\rho^{3 / 2}}{\pi} \frac{x_{\mu} \bar{\sigma}_{\mu \dot{\alpha} \alpha}}{\left(x^{2}\right)^{1 / 2}\left(x^{2}+\rho^{2}\right)^{3 / 2}} .
$$

The next step is to Fourier transform (4.2) to momentum space, remove the free fermion propagator $i p^{\alpha \dot{\alpha}} / p^{2}$ and go on mass-shell,

$$
\lim _{p^{2} \rightarrow 0} i p^{\alpha \dot{\alpha}} \tilde{\psi}_{\dot{\alpha} \beta}^{(0)}(p)=2 \pi \rho^{3 / 2} .
$$

Combining this instanton LSZ-reduced fermion zero mode contribution with the corresponding anti-instanton fermion zero mode, $\tilde{\psi}^{(0) \dagger}$, and including the external spinor factors we obtain the desired quark-line field insertion (for a single incoming fermion) to the forward elastic scattering amplitude in the instanton-anti-instanton background:

$$
\operatorname{Tr}\left(\tilde{\psi}^{(0) \dagger} \tilde{\psi}^{(0)}\right)=4 \pi^{2} \rho^{3 / 2} \bar{\rho}^{3 / 2}(p \cdot \hat{R}) e^{i R \cdot p},
$$

where we have restored the instanton-anti-instanton separation $R$ and the relative instanton-anti-instanton orientation matrix, in the same way as explained in the paragraph below Eq. (2.11). We now analytically continue to Minkowski space and further generalize the expression above to allow for a nonzero virtuality $Q$ of the quark momentum, as we did earlier for the gluon in (2.13). This gives

$$
\frac{1}{\omega_{\text {ferm }}} \operatorname{Tr}\left(\tilde{\psi}^{(0) \dagger} \tilde{\psi}^{(0)}\right)=4 \pi^{2} \sqrt{\hat{s}} \rho^{3 / 2} \bar{\rho}^{3 / 2} J(Q \rho) J(Q \bar{\rho}) e^{R \cdot p} \frac{1}{\omega_{\text {ferm }}},
$$


where the form factors $J(Q \rho)$ and $J(Q \bar{\rho})$ are the same functions $[21,22]$ as in the gluon case in (2.14). Note that we have also included in (4.5) the factor of $1 / \omega_{\text {ferm }}$, where $\omega_{\text {ferm }}$ is the fermionic action term computed on the antiinstanton and instanton zero modes,

$$
\omega_{\mathrm{ferm}}=\int d^{4} x \tilde{\psi}^{(0) \dagger}(x) i \not D \tilde{\psi}^{(0)}(x),
$$

and is computed in (A8). If $2 N_{f}$ fermions were present in the final state, such as in the original two-gluon initiated process,

$$
g+g \rightarrow n_{g} \times g+\sum_{f=1}^{N_{f}}\left(q_{R f}+\bar{q}_{L f}\right),
$$

they would contribute to the total cross section a factor of $\mathcal{K}_{\text {ferm }}=\left(\omega_{\text {ferm }}\right)^{2 N_{f}}$, i.e., one factor of $\omega_{\text {ferm }}$ for each fermion/antifermion in the final state, which is precisely what we have in Eqs. (2.5) and (2.6). In the present case, however, when a fermion is present in the initial state, we have one less fermion in the final state,

$$
\begin{gathered}
q_{L}+g \rightarrow n_{g} \times g+q_{R}+\sum_{f=1}^{N_{f}-1}\left(q_{R f}+\bar{q}_{L f}\right), \\
\bar{q}_{R}+g \rightarrow n_{g} \times g+\bar{q}_{L}+\sum_{f=1}^{N_{f}-1}\left(q_{R f}+\bar{q}_{L f}\right) .
\end{gathered}
$$

This implies that we have to correct the $\mathcal{K}_{\text {ferm }}$ factor in the instanton cross section for (4.8) or (4.9) by including a $1 / \omega_{\text {ferm }}$ factor in the initial-state fermion line insertion. This is what we have done in (4.5). We also note that there are $N_{f}$ processes of the type (4.8) and the same for the processes (4.9) initiated by the antiquark. Instanton fermion zero mode expressions are the same for $q_{L}$ and $\bar{q}_{R}$; hence there is essentially an additional enhancement factor of $2 N_{f}$ to be included in (4.5) counting all light quarks and antiquarks in the initial state.

We can now comment on the relative effect of having an incoming fermion versus an incoming gluon by taking the ratio of the expressions on the right-hand sides of (4.5) and (2.13). This gives the relative suppression factor of

$$
\frac{\alpha_{s}}{\rho \sqrt{\hat{s}}} \frac{16}{\pi} \frac{1}{\omega_{\text {ferm }}} \propto \frac{16}{\pi} \alpha_{s} \frac{1}{\langle\rho\rangle E},
$$

which using the data from Table 1 in [11] gives

0.11 for the instanton mass $E=30 \mathrm{GeV}$,

0.086 for the instanton mass $E=50 \mathrm{GeV}$,

0.063 for the instanton mass $E=100 \mathrm{GeV}$.

For the partonic instanton cross sections for the process in Fig. 3(a) we use one insertion of (4.5) and one insertion of (2.13). Also there is an $\alpha_{e m}^{2}$ in the cross section coming from the emission and the splitting of the photon. For Fig. 3(b) we use one insertion of (4.5) and one insertion of (2.21). And we have $\alpha_{e m}^{2}$ as above. For Fig. 3(c) we use two insertions of (4.5). Also since there are two photons we have an additional $\alpha_{e m}^{4}$ in the cross section.

\section{B. Physical photon induced cross sections}

The photon flux in the LO approximation is

$$
\frac{d n_{\gamma}}{d x}=\frac{\alpha_{e m}}{x \pi} \int_{\left(x m_{p}\right)^{2}} \frac{d q^{2}}{q^{2}} F_{N}^{2}\left(q^{2}\right)=\frac{\alpha_{e m}}{x \pi} 2 \ln \left(\frac{1}{x m_{p} R}\right),
$$

where $\alpha_{e m}=1 / 137$ is the QED coupling, $m_{p}$ is the proton mass, and $F_{N}\left(q^{2}\right)$ is its form factor; $R^{2} \sim 5-6 \mathrm{GeV}^{-2}$ is the slope of $F_{N}^{2}\left(q^{2}\right)$.

The photon does not interact with the gluon and the quark photon vertex conserves the helicity of light quarks. Therefore there are no parton loops from the photon side. Recall that the light quark zero mode in the instanton field contains a quark and an antiquark of different helicities (such as $q_{L} \bar{q}_{R}$ ). Thus we have to consider the three diagrams of Fig. 3 only.

An effective unintegrated quark distribution produced by the photon reads

$$
F_{i}^{\gamma}(z, Q)=\frac{\alpha_{e m}}{2 \pi} e_{i}^{2} T_{i}(Q, \bar{\mu})\left(z^{2}+(1-z)^{2}\right),
$$

where $e_{i}$ is the electric charge of quark $i$ and $T_{i}$ is given by (3.7).

Thus the Fig. 3 cross sections can be written as

$$
\begin{aligned}
\sigma^{(3 a)}= & \frac{d n_{\gamma}}{d x_{\gamma}} \int \sum_{i j} \frac{d x_{\mathbb{P}} d z_{1} d z_{2}}{x_{\mathbb{P}} z_{1}} d x_{\gamma} \cdot \int F_{i}^{D}\left(x_{\mathbb{P}}, z_{1}, Q_{1 t}\right) \\
& \times F_{j}^{\gamma}\left(z_{2}, Q_{2 t}\right) \hat{\sigma}_{i j}\left(\hat{s}, Q_{1 t}, Q_{2 t}\right) \frac{d Q_{1 t}^{2} d Q_{2 t}^{2}}{Q_{1 t}^{2} Q_{2 t}^{2}}, \\
\sigma^{(3 b)}= & \frac{d n_{\gamma}}{d x_{\gamma}} \int \sum_{i} \frac{d z_{1} d x_{2}}{x_{2}} d x_{\gamma} \\
& \times\left[\int \frac{d Q_{2 t}^{2}}{Q_{2 t}^{2}} R_{2 g} F_{2 g}\left(x_{2}, Q_{2 t} ; t_{2}\right)\right]^{2} d t_{2} \\
& \cdot \frac{1}{16 \pi^{2}} \int F_{j}^{\gamma}\left(z_{1}, Q_{1 t}\right) \hat{\sigma}_{i g}\left(\hat{s}, Q_{1 t}, Q_{2 t}\right) \frac{d Q_{1 t}^{2}}{Q_{1 t}^{2}}, \quad(4.14) \\
\sigma^{(3 c)}= & \frac{d n_{1 \gamma}}{d x_{1 \gamma}} \frac{d n_{2 \gamma}}{d x_{2 \gamma}} \int \sum_{i j} d z_{1} d z_{2} d x_{1 \gamma} d x_{2 \gamma} \\
& \cdot \int F_{i}^{\gamma}\left(z_{1}, Q_{1 t}\right) F_{j}^{\gamma}\left(z_{2}, Q_{2 t}\right) \hat{\sigma}_{i j}\left(\hat{s}, Q_{1 t}, Q_{2 t}\right) \frac{d Q_{1 t}^{2} d Q_{2 t}^{2}}{Q_{1 t}^{2} Q_{2 t}^{2}} .
\end{aligned}
$$


TABLE I. Instanton cross sections at the $14 \mathrm{TeV}$ LHC. The differential cross sections for the process in Figs. 1(a),1(b) and 2(a), 2(b), given by Eqs. (5.1) and (5.2), are computed for a range of instanton masses $M_{\text {inst }}$.

\begin{tabular}{llllll}
\hline \hline$M_{\text {inst }}[\mathrm{GeV}]$ & $d \sigma_{p p}^{(1 a)}[\mathrm{pb}]$ & $d \sigma_{p p}^{(1 b)}[\mathrm{pb}]$ & $d \sigma_{p p}^{(2 a)}[\mathrm{pb}]$ & $d \sigma_{p p}^{(2 b)}[\mathrm{pb}]$ & $d \sigma_{p p}^{(2 b)}, Q_{t}>20 \mathrm{GeV}$ \\
\hline 15 & 13.3 & $4.56 \times 10^{4}$ & $3.72 \times 10^{3}$ & $1.83 \times 10^{5}$ & $\ldots$ \\
35 & $6 \times 10^{-3}$ & $1.69 \times 10^{2}$ & 8.10 & $2.28 \times 10^{3}$ & $1.99 \times 10^{-3}$ \\
55 & $3.82 \times 10^{-5}$ & 3.27 & $1.19 \times 10^{-1}$ & $8.96 \times 10^{1}$ & $2.95 \times 10^{-3}$ \\
75 & $8.8 \times 10^{-7}$ & $1.61 \times 10^{-1}$ & $4.72 \times 10^{-3}$ & 7.06 & $1.70 \times 10^{-3}$ \\
95 & $4.27 \times 10^{-8}$ & $1.38 \times 10^{-2}$ & $3.42 \times 10^{-4}$ & $8.58 \times 10^{-1}$ & $7.26 \times 10^{-4}$ \\
115 & $3.37 \times 10^{-9}$ & $1.74 \times 10^{-3}$ & $3.68 \times 10^{-5}$ & $1.39 \times 10^{-1}$ & $2.80 \times 10^{-4}$ \\
135 & $3.77 \times 10^{-10}$ & $2.86 \times 10^{-4}$ & $5.29 \times 10^{-6}$ & $2.75 \times 10^{-2}$ & $1.04 \times 10^{-4}$ \\
\hline \hline
\end{tabular}

Here we neglect the gap survival factor since the photon exchange occurs at large impact parameters where $S^{2}$ is close to $1[33,34]$.

\section{RESULTS}

As was shown in Sec. IVA the instanton production cross section induced by quarks is an order of magnitude suppressed in comparison with that induced by gluons. The processes where the energy across the LRG is transferred by a photon instead of the Pomeron are additionally suppressed by the QED coupling $\alpha_{e m}^{2} \sim 10^{-4}$. Therefore below we will consider the central instanton production caused by the gluon component of the Pomeron only. ${ }^{15}$

As in [11] we have used the diffractive PDF given by the $\mathrm{H} 1$ fit B [23] and for the inclusive PDFs we use the NNPDF 3.1 NNLO set with $\alpha_{s}\left(M_{Z}\right)=0.118$ [37]. For the instanton part the one loop running QCD coupling $\alpha_{s}(\mu)$ was normalized at the $\tau$ mass, $\alpha_{s}\left(m_{\tau}\right)=0.32$, and was frozen at $\alpha_{s}=0.35$ at $\mu<1.45 \mathrm{GeV}$ (see [11] for more details).

The gap survival factor was taken to be $S^{2}=0.05-$ twice smaller than that in [11]. While in [11] we consider the single dissociation (one LRG), here we deal with the central diffractive process (with two LRGs). As was shown in, e.g., [38] (see also [39]) in this case the expected gap survival probability is about 2 times smaller due to a lower typical impact parameter, $b_{t}$.

Our results are presented in Table I for the LHC $p p$ energy $\sqrt{s}_{p p}=14 \mathrm{TeV}$. Specifically, for the central instanton production processes shown in Figs. 1(a) and 1(b), the second and the third columns in Table 1 present the differential cross sections $d \sigma^{(1 a)}$ and $d \sigma^{(1 b)}$ defined via

$$
\begin{aligned}
(1 a) \&(1 b): d \sigma & =d \sigma /\left(d \ln \left(x_{\mathbb{P} 1}\right) d \ln \left(x_{\mathbb{P} 2}\right)\right) \\
& =d \sigma /\left(d \ln \left(M_{\mathrm{inst}}^{2}\right) d Y\right),
\end{aligned}
$$

\footnotetext{
${ }^{15}$ Note that photon-mediated processes could be of special interest in the case of heavy ion ultraperipheral collisions, where the flux of emitted photons is enhanced by a factor $Z^{2}$ in comparison to the proton case, where $Z$ is the ion charge number (see, for example, [35,36]). Thus the instanton production in heavy ion collisions needs an additional study.
}

at $\quad x_{\mathbb{P} 1}=x_{\mathbb{P} 2}=M_{\text {inst }} / \sqrt{s_{p p}}$. This corresponds to the instanton rapidity $Y=0$ and, as always, the instanton mass $M_{\text {inst }}$ denotes the c.m. partonic energy entering the instanton vertex, $M_{\text {inst }}:=\sqrt{\hat{s}}$.

For the case of the 2(a) and 2(b) processes, the corresponding differential cross sections $d \sigma^{(2 a)}$ and $d \sigma^{(2 b)}$ shown in the last three columns of Table I are

$(2 a) \&(2 b): d \sigma=d \sigma /\left(d \ln \left(x_{\mathbb{P} 1}\right) d \ln \left(x_{\mathbb{P} 2}\right) d \ln \left(M_{\text {inst }}^{2}\right) d Y\right)$,

at $x_{\mathbb{P} 1}=x_{\mathbb{P} 2}=0.03$ integrated over $z$. This value of $x_{\mathbb{P}}$ corresponds to the characteristic region of the PPS and AFP detectors.

The differential cross sections (5.1) and (5.2) depend on the instanton mass, and Table I shows our predictions for different values of $M_{\text {inst }}$.

As is seen from Table I the contribution of diagrams Fig. 1(a) [2(a)], where both gluons created by the Pomeron couple to the instanton, is much smaller than that from Fig. 1(b) [2(b)]. This is explained by the fact that we have the elementary gluon pair insertion $(2.21),\langle\cdots\rangle_{g g} \propto Q_{t}^{2} \bar{Q}_{t}^{2}$ instead of the much larger factor $M_{\text {inst }}^{4}$ in the single gluon case (2.16), $\langle\cdots\rangle_{g+g} \propto \hat{s}^{2}=M_{\text {inst }}^{4}$.

Next we can see that the pure exclusive Fig. 1(b) cross section is suppressed in comparison with the Fig. 2(b) case where we allow the radiation of "spectator" jet(s). The suppression is mainly caused by the Sudakov-like $T$-factor (3.7) and becomes stronger for a larger $M_{\text {inst }}$.

We will now argue that our findings amount to large and potentially observable instanton cross sections at the LHC. It was expected from the outset that the probability to centrally produce an instanton of not too low mass would be rather small. Our data show that for $M_{\text {inst }}>50 \mathrm{GeV}$ this ultimately translates into picobarn-level cross sections in the exclusive case and hundreds of pb for the Fig. 2(b) configuration. However, for the high-luminosity LHC runs these predictions listed in Table I can, in fact, be interpreted as sufficiently large effects. To give a rough estimate, the differential cross sections (5.1) and (5.2) in Table I should be multiplied by the available intervals in $\ln \left(x_{\mathbb{P}}\right) \sim 1-2$, in 
$\ln M_{\text {inst }}^{2} \sim 2-4$, and in $\Delta Y \sim 2-4$ roughly giving an extra order of magnitude. This enhancement is expected to be lost after accounting for the detector acceptance and imposing relevant kinematic cuts. The integrated luminosity expected at the LHC high-luminosity runs is up to $L=30001 / \mathrm{fb}$. That is, even in the pessimistic scenario with $\sigma=10^{-4} \mathrm{pb}$ we expect 300 events. For a more optimistic case of $\sigma \sim \mathrm{pb}$ we have orders of magnitude more events (though not accounting for background). Properly accounting for large background from soft QCD deserves a separate discussion that goes beyond the scope of the present paper and that we plan to address in the future [40].

We end this section with some additional comments. We note that it is quite challenging to select pure exclusive events. Most probably it will be impossible to distinguish the jet radiated off the instanton from the "spectator" jet. That is, we will deal mainly with the Fig. 2(b) configuration and without additional cuts the major contribution will come from the low mass $\left(M_{\text {inst }}\right)$ semi-inclusive events. On the other hand, the theoretical accuracy of the predictions for low mass instantons is less reliable. Moreover, it will be hard to discriminate between the low mass instanton and the relatively soft underlying events.

To select a reasonably large $M_{\text {inst }}$ we have to introduce additional cuts such as those proposed in [11], say, to ask for sufficiently large multiplicity and the sum of transverse energies, $\sum_{i} E_{T i}$ of the secondaries in some limited central rapidity interval. These cuts will additionally reduce the expected cross section up to an order of magnitude.

An alternative way to suppress small $M_{\text {inst }}$ is to enlarge the virtuality, $Q^{2}$, of the incoming gluon. Then the form factor $J(Q \rho)$ will select only the small size (i.e., sufficiently large mass) instantons. This idea was proposed in [41] for deep inelastic scattering and was considered in [9] for the case of proton-proton collisions. That is, we have to consider the events with a rather large transverse momentum of spectator jet. The corresponding cross sections are shown in the last column of Table I. It is seen that the $Q_{t}>20 \mathrm{GeV}$ cut strongly suppresses the low $M_{\text {inst }} \lesssim$ $150 \mathrm{GeV}$ contribution. The resulting cross section in this case $\sim 10^{-4} \mathrm{pb}$ becomes quite small which puts these processes at a significant disadvantage relative to the processes in column 4 of Table I.

\section{CONCLUSION}

We have considered how to produce the QCD instanton in the central rapidity region in proton-proton collisions at the LHC. These are events where the produced instanton is separated from the two original protons on both sides by two large rapidity gaps. We showed that the cross section for pure exclusive processes in Fig. 1 is strongly suppressed in comparison with the processes in Fig. 2 in which the radiation of spectator jets is allowed.
The dominant contribution comes from the diagrams where only one gluon from each Pomeron couples to the instanton. The configurations where the energy needed to create the instanton is transferred through the quark line is also suppressed by about an order of magnitude. For a large instanton mass these suppressions become stronger.

The photon induced QED contribution, shown in Fig. 3, where the Pomeron in an LRG interval is replaced by the photon, is negligible due to the quark suppression and a very small QED coupling $\alpha_{e m}^{2}$.

For a reasonable instanton mass $M_{\text {inst }} \gtrsim 50 \mathrm{GeV}$ the expected cross sections for Pomeron-mediated central instanton production are of the order of picobarns in the pure exclusive case and increase up to hundreds of $\mathrm{pb}$ when we allow the emission of spectator jets. These signal cross sections are encouragingly large and assuming that backgrounds are manageable and can be accounted for in the future, there is a tantalizing chance that QCD instanton effects can be either seen or ruled out in central processes at the LHC.

As shown in [11], the kinematic regime with large rapidity gaps offers a good possibility of detecting instantons as the signal events outnumber the background events significantly. One may compare this approach to that of [9] where the requirement of large rapidity gaps was not imposed. In this approach the signal events do not outnumber the background events but there still remains the possibility of detecting the instanton. A first analysis of data was carried out in [10] along similar lines.

Moving to the regime with large rapidity gaps suppresses the instanton cross section quite heavily but the requirement of large rapidity gaps (along with other selection criteria) suppresses the background cross section even more leading to, on the whole, a cleaner experimental signature with potentially better prospects of detection [11]. As shown in this paper the requirement of two large rapidity gaps suppresses the instanton cross section further but it may suppress the background even more as was shown for the case of one large rapidity gap. Although we pay the price of a lower instanton cross section, this would not be such a great concern at the high luminosity LHC where even for $\sim \mathrm{fb}$ cross sections we would expect thousands of signal events.

\section{ACKNOWLEDGMENTS}

We thank Marek Tasevsky for useful discussions. The research of V. V. K. is supported by the UK Science and Technology Facilities Council (STFC) under Grant No. ST/P001246/1, and D. L. M. acknowledges an STFC studentship.

$$
\text { APPENDIX: } D(\rho), S_{\overline{I I}}(z) \text {, and } \mathcal{K}_{\text {ferm }}(z)
$$

The functions appearing in the instanton integrand for the cross section in (2.6) are given by known expressions 
and are taken from [9]. We collect them here for the reader's convenience.

We start with the instanton density [3]

$$
D\left(\rho, \mu_{r}\right)=\kappa \frac{1}{\rho^{5}}\left(\frac{2 \pi}{\alpha_{s}\left(\mu_{r}\right)}\right)^{2 N_{c}}\left(\rho \mu_{r}\right)^{b_{0}},
$$

where $\kappa$ is the normalization constant in the $\overline{\mathrm{MS}}$ scheme,

$$
\kappa=\frac{2 e^{5 / 6-1.511374 N_{c}}}{\pi^{2}\left(N_{c}-1\right) !\left(N_{c}-2\right) !} e^{0.291746 N_{f}} \simeq 0.0025 e^{0.291746 N_{f}} .
$$

The next function to specify is the instanton-anti-instanton bosonic action, $S_{I \bar{I}}(z)=\frac{4 \pi}{\alpha_{s}} \mathcal{S}(z)$, where

$$
\begin{aligned}
\mathcal{S}(z)= & 3 \frac{6 z^{2}-14}{(z-1 / z)^{2}}-17-3 \log (z) \\
& \times\left(\frac{(z-5 / z)(z+1 / z)^{2}}{(z-1 / z)^{3}}-1\right),
\end{aligned}
$$

which is a function of a single variable $z$ known as the conformal ratio of the (anti)-instanton collective coordinates $[42,43]$,

$$
z=\frac{R^{2}+\rho^{2}+\bar{\rho}^{2}+\sqrt{\left(R^{2}+\rho^{2}+\bar{\rho}^{2}\right)^{2}-4 \rho^{2} \bar{\rho}^{2}}}{2 \rho \bar{\rho}} .
$$

The fermionic factor $\mathcal{K}_{\text {ferm }}$ is

$$
\mathcal{K}_{\text {ferm }}=\left(\omega_{\text {ferm }}\right)^{2 N_{f}},
$$

where

$$
\omega_{\mathrm{ferm}}=\int d^{4} x \tilde{\psi}^{(0) \dagger}(x) i \not D \tilde{\psi}^{(0)}(x)
$$

is the fermionic action term computed on the anti-instanton and instanton fermion zero modes. For a process, such as

$$
g+g \rightarrow n_{g} \times g+\sum_{f=1}^{N_{f}}\left(q_{R f}+\bar{q}_{L f}\right)
$$

where there are $2 N_{f}$ fermions present in the final state we need to saturate $2 N_{f}$ grassmanian integrations over instanton and anti-instanton fermion zero modes. This brings down the fermionic action term $\omega_{\text {ferm }}$ to the integration measure $2 N_{f}$ times, which is reflected in (A5).

For $\omega_{\text {ferm }}$ we use the expression derived in [41]

$$
\omega_{\text {ferm }}(z)=\frac{3 \pi}{8} \frac{1}{z^{3 / 2}} 2 F_{1}\left(\frac{3}{2}, \frac{3}{2} ; 4 ; 1-\frac{1}{z^{2}}\right)
$$

as a function of the $z$-variable (A4).

Finally, as in the earlier works $[9,11]$, the renormalization scale in (A1) and for the running coupling $\alpha_{s}$ is set to the inverse instanton size. This prescription removes the large $\left(\rho \mu_{r}\right)^{b_{0}}\left(\bar{\rho} \mu_{r}\right)^{b_{0}}$ factors from the instanton and antiinstanton densities $D(\rho)$ and $D(\bar{\rho})$. Hence we choose

$$
\mu_{r}=1 /\langle\rho\rangle=1 / \sqrt{\rho \bar{\rho}} .
$$

For the reference point of $\alpha_{s}$ we choose its value at the $\tau$ mass, as explained in [11],

$$
\frac{4 \pi}{\alpha_{s}(\langle\rho\rangle)} \simeq \begin{cases}\frac{4 \pi}{0.32}-2 b_{0} \log \left(\langle\rho\rangle m_{\tau}\right) & : \text { for }\langle\rho\rangle^{-1} \geq 1.45 \mathrm{GeV}, \\ \frac{4 \pi}{0.35} & \text { :for }\langle\rho\rangle^{-1}<1.45 \mathrm{GeV} .\end{cases}
$$

At these energy scales we are in the regime of

$$
N_{f}=4
$$

active quarks, and this is the $N_{f}$ value we use in $b_{0}=$ $11-2 N_{f} / 3$ and in the instanton density expressions for $\kappa$ and $\mathcal{K}_{\text {ferm }}$ in (A5).
[1] A. A. Belavin, A. M. Polyakov, A. S. Schwartz, and Y. S. Tyupkin, Pseudoparticle solutions of the Yang-Mills equations, Phys. Lett. 59B, 85 (1975).

[2] G. 't Hooft, Computation of the quantum effects due to a four-dimensional pseudoparticle, Phys. Rev. D 14, 3432 (1976); Erratum, Phys. Rev. D 18, 2199 (1978).

[3] G. 't Hooft, How instantons solve the U(1) problem, Phys. Rep. 142, 357 (1986).
[4] A. I. Vainshtein, V. I. Zakharov, V. A. Novikov, and M. A. Shifman, ABC's of instantons, Usp. Fiz. Nauk 136, 553 (1982) [Sov. Phys. Usp. 25, 195 (1982)].

[5] T. Schäfer and E. V. Shuryak, Instantons in QCD, Rev. Mod. Phys. 70, 323 (1998).

[6] N. Dorey, T. J. Hollowood, V. V. Khoze, and M. P. Mattis, The calculus of many instantons, Phys. Rep. 371, 231 (2002). 
[7] I. I. Balitsky and M. G. Ryskin, The possibility of experimental observation of the QCD instanton, Yad. Fiz. 56N8, 196 (1993) [Phys. At. Nucl. 56, 1106 (1993)]; The possibility to observe QCD instanton, Phys. Lett. B 296, 185 (1992).

[8] V. V. Khoze, F. Krauss, and M. Schott, Large effects from small QCD instantons: Making soft bombs at hadron colliders, J. High Energy Phys. 04 (2020) 201.

[9] V. V. Khoze, D. L. Milne, and M. Spannowsky, Searching for QCD instantons at hadron colliders, Phys. Rev. D 103, 014017 (2021).

[10] S. Amoroso, D. Kar, and M. Schott, How to discover QCD instantons at the LHC, Eur. Phys. J. C 81, 624 (2021).

[11] V. A. Khoze, V. V. Khoze, D. L. Milne, and M. G. Ryskin, Hunting for QCD instantons at the LHC in events with large rapidity gaps, Phys. Rev. D 104, 054013 (2021).

[12] V. A. Khoze, A. D. Martin, and M. G. Ryskin, Prospects for new physics observations in diffractive processes at the LHC and Tevatron, Eur. Phys. J. C 23, 311 (2002).

[13] E. Shuryak and I. Zahed, Semiclassical double pomeron production of glueballs and eta-prime, Phys. Rev. D 68, 034001 (2003).

[14] S. Abdel Khalek, B. Allongue, F. Anghinolfi, P. Barrillon, G. Blanchot, S. Blin-Bondil, A. Braem, L. Chytka, P. Conde Muíño, M. Düren et al., The ALFA roman pot detectors of ATLAS, J. Instrum. 11, P11013 (2016).

[15] G. Anelli et al. (TOTEM Collaboration), The TOTEM experiment at the CERN large hadron collider, J. Instrum. 3, S08007 (2008).

[16] The AFP project in ATLAS, Letter of intent of the phase-I upgrade (ATLAS Collabration), http://cdsweb.cern.ch/ record/1402470.

[17] The CMS and TOTEM Collaborations, CMS-TOTEM precision proton spectrometer technical design report, Report Nos. CERN-LHCC-2014-021, TOTEM-TDR-003, CMS-TDR-13, 2014.

[18] The CMS Collaboration, The CMS precision proton spectrometer at the HL-LHC-expression of interest, arXiv: 2103.02752.

[19] A. H. Mueller, First quantum corrections to gluon-gluon collisions in the one instanton sector, Nucl. Phys. B348, 310 (1991); Leading power corrections to the semiclassical approximation for gauge meson collisions in the one instanton sector, B353, 44 (1991).

[20] I. I. Balitsky and V. M. Braun, Instanton induced contributions to structure functions of deep inelastic scattering, Phys. Lett. B 314, 237 (1993).

[21] S. Moch, A. Ringwald, and F. Schrempp, Instantons in deep inelastic scattering: The Simplest process, Nucl. Phys. B507, 134 (1997).

[22] A. Ringwald, Electroweak instantons/sphalerons at VLHC? Phys. Lett. B 555, 227 (2003).

[23] A. Aktas et al. (H1 Collaboration), Measurement and QCD analysis of the diffractive deep-inelastic scattering crosssection at HERA, Eur. Phys. J. C 48, 715 (2006).
[24] M. Botje, QCDNUM: Fast QCD evolution and convolution, Comput. Phys. Commun. 182, 490 (2011).

[25] M. A. Kimber, A. D. Martin, and M. G. Ryskin, Unintegrated parton distributions, Phys. Rev. D 63, 114027 (2001).

[26] J. Collins and T.C. Rogers, Connecting different TMD factorization formalisms in QCD, Phys. Rev. D 96, 054011 (2017).

[27] V. Saleev, Production of three isolated photons in the highenergy factorization approach, arXiv:2107.11147.

[28] B. A. Kniehl, D. V. Vasin, and V. A. Saleev, Charmonium production at high energy in the $k_{T}$-factorization approach, Phys. Rev. D 73, 074022 (2006).

[29] T. D. Coughlin and J. R. Forshaw, Central exclusive production in QCD, J. High Energy Phys. 01 (2010) 121.

[30] V. A. Khoze, A. D. Martin, and M. G. Ryskin, Can the Higgs be seen in rapidity gap events at the Tevatron or the LHC? Eur. Phys. J. C 14, 525 (2000).

[31] A. G. Shuvaev, K. J. Golec-Biernat, A. D. Martin, and M. G. Ryskin, Off diagonal distributions fixed by diagonal partons at small x and xi, Phys. Rev. D 60, 014015 (1999).

[32] A. Shuvaev, Solution of the off forward leading logarithmic evolution equation based on the Gegenbauer moments inversion, Phys. Rev. D 60, 116005 (1999).

[33] V. A. Khoze, A. D. Martin, R. Orava, and M. G. Ryskin, Luminosity monitors at the LHC, Eur. Phys. J. C 19, 313 (2001).

[34] V. A. Khoze, A. D. Martin, and M. G. Ryskin, Photon exchange processes at hadron colliders as a probe of the dynamics of diffraction, Eur. Phys. J. C 24, 459 (2002).

[35] R. Bruce, D. d'Enterria, A. de Roeck, M. Drewes, G. R. Farrar, A. Giammanco, O. Gould, J. Hajer, L. Harland-Lang, J. Heisig et al., New physics searches with heavy-ion collisions at the CERN Large Hadron Collider, J. Phys. G 47, 060501 (2020).

[36] L. A. Harland-Lang, V. A. Khoze, and M. G. Ryskin, Exclusive LHC physics with heavy ions: SuperChic 3, Eur. Phys. J. C 79, 39 (2019).

[37] V. Bertone et al. (NNPDF Collaboration), Illuminating the photon content of the proton within a global PDF analysis, SciPost Phys. 5, 008 (2018).

[38] V. A. Khoze, A. D. Martin, and M. G. Ryskin, Soft diffraction and the elastic slope at Tevatron and LHC energies: A MultiPomeron approach, Eur. Phys. J. C 18, 167 (2000).

[39] V. A. Khoze, A. D. Martin, and M. G. Ryskin, Multiple interactions and rapidity gap survival, J. Phys. G 45, 053002 (2018).

[40] M. Tasevsky et al. (to be published).

[41] A. Ringwald and F. Schrempp, Instanton induced crosssections in deep inelastic scattering, Phys. Lett. B 438, 217 (1998).

[42] A. V. Yung, Instanton vacuum in supersymmetric QCD, Nucl. Phys. B297, 47 (1988).

[43] V. V. Khoze and A. Ringwald, Nonperturbative contribution to total cross-sections in non-Abelian gauge theories, Phys. Lett. B 259, 106 (1991); Valley trajectories in gauge theories, Report No. CERN-TH-6082-91, 1991. 